\title{
移动计算环境下不确定性组合服务的事务建模与 执行分析
}

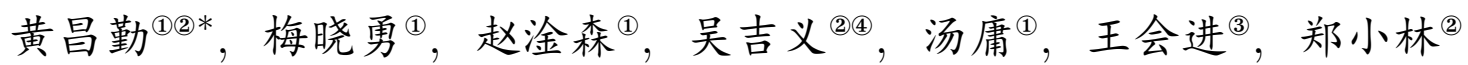

(1) 华南师范大学信息服务软件技术研究中心, 广州 510631

(2) 浙江大学电子服务研究中心, 杭州 310027

(3) 暨南大学计算机系, 广州 510632

(4) 杭州师范大学电子商务与信息安全重点实验室, 杭州 310036

* 通信作者. E-mail: cqhuang@zju.edu.cn

收稿日期: 2014-09-18; 接受日期: 2014-11-12

国家自然科学基金 (批准号: 61370229, 61370178, 61272067)、国家科技支撑计划 (批准号: 2013BAH72B01)、广东省自然科学基 金 (批准号: S2013010015178)、广东省科技计划 (批准号: 2012A032200018)、中国博士后科学基金 (批准号: 2014M562188) 和广 东省教育厅科技创新 (批准号: 2012KJCX0037) 资助项目

摘要＼cjkstart移动计算环境中, 服务面临资源受限与连接波动, 同时存在越区切换和弱一致性需求等事务 性挑战. 为了确保移动环境下组合服务的事务有效性, 本文以服务的不确定性和事务属性为基础, 提 出了一种基于 Markov 链的移动组合事务模型。首先通过形式化方法构建移动环境下原子服务模型 和原子服务的事务机制, 并基于状态概率实现了原子服务的 2 维不确定性表征, 然后运用 Markov 链 完成移动组合服务的模型表达, 提出了该模型下的不确定性处理与事务方法, 最后基于典型业务结 构分析了移动组合事务的执行语义与实施. 实验表明: 该事务模型能有效描述移动环境下组合服务 的事务需求, 且服务不确定性的不同指标和各事务架构皆对组合事务成功率产生较大影响.

关键词 组合服务 不确定性依赖 Markov 链 事务 执行分析

\section{1 引言}

移动服务计算是移动互联网和云计算的重要支持技术, 由于其服务的便捷性和计算资源共享的泛 在性, 它为网络技术发展带来了新机遇, 同时也面临诸多挑战, 其中因资源受限与环境波动带来的服务 高度不确定性是必须克服的困难之一 ${ }^{[1]}$. 面对移动业务流, 如何确保组合服务可信执行与如何基于组 合服务建构有效的事务模型, 成为了移动环境下组合事务可靠执行的关键 ${ }^{[2]}$.

原子性和一致性是事务研究的核心问题, 在服务计算环境下, 基于服务组合的事务必定是组合事 务, 考虑移动因素, 事务机制必须适应信道切换, 由此必须解决原子服务与组合服务在移动计算环境下 的失败问题、补偿机制及其相关验证问题 (含形式化验证与应用测试). 在移动计算环境中, 无论原子 服务还是组合服务, 它们都至少面临以下三方面的挑战 ${ }^{[3]}$ : (1) 移动设备资源位置变动性; (2) 移动设 备资源与网络连接的高频率不稳定性; (3) 移动设备资源的特殊性 (如较低的计算力和电能等). 在移 
动计算环境下不确定性组合服务的事务研究, 必将考虑情境因素的不确定性和组合事务的特殊性, 解 决模型、验证和可信保障等问题.

本文拟以原子服务为基础, 充分考虑上述三方面的不确定性因素, 从模型上表征服务的不确定性, 然后以业务流分析为基础, 运用 Markov 链建立移动组合服务的事务模型, 最后在模型中完成不确定 性聚合和特性分析, 并建构典型组合事务处理机制, 在考虑不确定性的同时满足事务处理需求.

\section{2 相关工作}

移动计算中组合事务研究涉足多方因素, 主要包括服务计算环境下的组合事务、移动计算环境下 的服务不确定性与不确定性组合服务的事务研究三方面.

在基于服务的组合事务研究方面, 多集中在组合事务模型与表达、基于恢复的一致性保障 (含补 偿) 等方向. 在协议方面, 大多协议或规范 (如 WS4BPEL, WSCI, WS-CDL) 所提供的事务处理策略 都太单一, 通常设计执行补偿任务来消除失败发生. 在补偿和恢复操作方面, 通常在范围 (WS4BPEL) 或上下文 (WSCI) 或编排 (WS-CDL) 层次上进行静态定义, 当范围或上下文发生变化时, 将导致重复 定义和额外工作 ${ }^{[4]}$, 其他工作如: Khachana 等 ${ }^{[5]}$ 提出了一个新的 Web 事务模型, 其核心是将事务的 ACID 特性进行自适性松弛; Kokash 与 Arbab ${ }^{[6]}$ 提出了一种事务型行为模型框架的同时, 采用了基于 通道的协调语言 Reo 来建模终止和补偿处理, 并将 LRTs 表达为 LTL 和类 CTL 逻辑, 运用模型检测 技术验证 LRTs. Rajaram 等 ${ }^{[7]}$ 针对商业事务要求提出一个针对原子服务的抽象机制, 使其可进行服 务的恢复属性描述, 并将事务型 Web 服务根据恢复代价类化为不同的恢复等级. 在建模与验证方面, 相关研究 ${ }^{[8]}$ 通常考虑组合事务的语法/流程正确性、行为兼容性、行为可替换性、语义一致性、恢复 策略正确性和事务执行一致性, 采用的理论模型有有限状态机、过程代数和 Petri 网等. 典型工作如: Zhang 等 ${ }^{[9]}$ 基于情境感知进程网络 $(\mathrm{CAPN})$ 提出一个感知 Web 服务交互模型, 其中模型的行为语义 借助通信系统进程代数予以描述, 这使得外部情境改变能较好反映于商务行为之上; Medjahed 等 [10] 提出了一个面向组合服务的自底向上故障模型, 并设计适应性的软状态协议以完成在组件服务中的异 常、缺陷处理或者改变扩散; Sun 等 $[11,12]$ 针对协作商业事务的不协作中心和事务动态问题, 先后设计 了 CoBTx-Net 和 BTx-Net 模型, 进行了可靠性和一致性保障与理论验证; 由于在支持计算机辅助验 证上 Petri 网比有限状态机和过程代数等提供了一个更宽基础支持, 支持如失败处理、补偿和恢复机 制等事务处理机制, 梅晓勇等 [13,14] 引入 Petri 网探讨 Web 服务组合的事务恢复机制, 构造支持 LRTs 的失败恢复算法, 分析典型控制结构的执行语义, 保障语义正确性. 从上述研究发现, 目前有较多形式 化方法建模组合服务事务, 但较少关注移动组合服务下的事务; 相关研究中虽有可信因素的考虑, 暂未 发现基于不确定性的组合事务研究.

在移动计算中服务的不确定性及其相关研究主要集中在移动计算中网络等因素的不确定性表达 和情境的不确定性建模. 在移动计算中各不确定性因素表达方面, Pezoa 等 [15] 提出了描述分布式计算 系统服务可靠性的刚性概率框架, 它运用差分微分方程组来刻画了具有随机服务和失效次数的网络节 点, 以及随机迟延的网络通信等情况, 并设计一个算法来执行优化以增强服务可靠性; Akkarajitsakul 等 [16] 较全面地论述了移动无线网络中位置和网络等因素的不确定性, 并介绍管理方法以提高可用性; Zhang 等 ${ }^{[17]}$ 针对无线射频识别网络和基于位置的服务网络中的不确定性, 提出了一个 U-Quadtree 索引结构, 建成了基于代价模型的有效不确定对象索引算法. 在整体环境建模方面, Lee 等 [18] 利用层 次性本体建模法描述普适上下文环境, 对本研究有较好的借鉴作用; Christian ${ }^{[19]}$ 提出了一个基于动态 上下文模型的质量感知性数据处理方法, 该方法首先基于关系代数对新代数和操作进行定义, 然后建 
模并处理不确定性新数据; Anagnostopoulos 等 [20] 为了支持判断用户的当前位置, 应用模糊理论来建 模普适计算环境中用户情境, 并基于模糊值进行上下文信息的不确定性推理; 莫同等 ${ }^{[21]}$ 基于情境感 知服务特征分析提出了一种情境感知服务系统框架. 这些研究虽然方法各异, 但皆限于环境上下文建 模, 尚未发现对移动环境中服务不确定性的系统研究成果.

在不确定性组合服务的事务研究方面, 目前研究成果稀少, 其主要相关研究局限于不确定性环境 下的服务组合问题, 如: Wen 等 ${ }^{[22]}$ 针对服务组合中 QoS 的实时波动性, 提出了一个不确定性概率领 衔的 top-k 服务机制, 通过设置领衔概率计算以选择恰当的原子服务, 该方案一定程度上缓解了组合 服务中的不确定性问题; 顾军等 ${ }^{[23]}$ 从服务故障与失效的角度, 研究组合服务中的服务可用性, 给出一 种考虑失效恢复的组合服务性能分析模型, 并采用排队 Petri 网进行描述, 研究实施重试和替换等稳 定性能的方法.

\section{3 移动环境下的原子服务模型}

\section{1 移动环境下原子服务假设}

在移动计算环境中, 原子服务本身、服务宿居环境以及服务对外通信链路的状况都会影响服务的 正常或正确执行. 在服务执行过程中, 无论是服务自身软体, 还是其支撑硬件环境发生故障或失效时, 服务也会因此损毁或者输出不可预测的结果. 目前大部分相关研究都是基于各事件不确定性相互独 立而又整体集成于一体为前提, 然后进行分析和建模的; 为了明晰主要关联, 本文对其做出如下基本 假设.

假设 (1): 移动计算环境中传统 (有线) 网络设备一般具有较高的可靠性, 并且其工作时间相对较 短, 可假定其设备在服务期间不会发生失效.

假设 (2): 当服务请求到达传统网络设备后, 传统网络设备对其能立即响应.

假设 (3): 原子服务的执行离不开 $\mathrm{CPU}$ 和内存.

假设 (4): 节点设备上的原子服务个数在考查时间段内服从齐次泊松 (Poisson) 分布.

假设 (5): 无线网络中的下行带宽 (速度) 和上行带宽 (速度) 相同并独立.

假设 (6): 节点各硬件和通信链路上的故障或失效过程在考查时间段内服从齐次泊松分布.

假设 (7): 原子服务构成软件体的失效过程在考查时间段内服从齐次泊松分布.

假设 (8)：原子服务宿居节点的各硬件、宿居的软件体、基于链路的对外数据交换的故障和失效 等事件相互独立.

假设 (9): 原子服务宿居节点设备的电能消耗量在其同一类型设备硬件状况下, 仅与节点设备的 正常工作时间长度成正比.

假设 (10): 原子服务对所宿居节点上的各硬件资源上的需求工作强度, 在考查时间段内服从对数 正态分布.

假设 (11): 原子服务在所宿居节点设备的移动过程中, 其与中心节点 (无线信号发射源) 的距离在 考查时间段内服从对数正态分布.

\section{2 支持事务属性的原子服务模型}

定义 $\mathbf{1}$ (服务宿居设备) 原子服务 $\mathrm{WS}_{a}$ 的宿居设备 $D$ 表示为六元组 $D=\left(T_{y}, C_{\mathrm{cpu}}, M_{\mathrm{in}}, M_{\mathrm{ex}}\right.$ ， $\left.P_{\mathrm{ow}}, L_{\mathrm{oc}}\right)$, 其中: 
- $T_{y}$ 表示服务宿居设备 $D$ 的设备类型, 它与设备的各性能指标密切相关. 如移动设备的类型不 同, 则其无线网络连接部件的发收信号增益不同.

- $C_{\mathrm{cpu}}$ 表示服务宿居设备 $D$ 的 $\mathrm{CPU}$, 是移动计算的核心部件.

- $M_{\mathrm{in}}$ 和 $M_{\mathrm{ex}}$ 分别表示服务宿居设备 $D$ 的内存和外存储器.

- $P_{\mathrm{ow}}$ 表示服务宿居设备 $D$ 的电能, 是移动计算设备工作的基础.

- $L_{\mathrm{oc}}$ 表示服务宿居设备 $D$ 在移动计算环境中的物理位置, 它和移动环境一起决定了移动计算设 备的网络通信质量. 在移动计算环境中拟用三维坐标 $(x, y, z)$ 表征.

定义 2 (原子服务) 原子服务 $\mathrm{WS}_{a}$ 可表示为一个七元组 $\mathrm{WS}_{a}=\left(E, S_{\mathrm{in}}, S_{\text {out }}, C, D, \mathbf{P}_{a}, \mathbf{P}_{r}\right)$, 其中:

- $E$ 表示原子服务的执行软件体及其内含数据, 该实体在单个计算节点 (移动或者固定节点) 上 完成任务执行.

- $S_{\text {in }}$ 表示原子服务的运行业务逻辑的输入参数集, 可为 $\emptyset$.

- $S_{\text {out }}$ 表示原子服务的运行业务逻辑的输出结果集, 可为 $\emptyset$.

- $C$ 表示原子服务的运行业务逻辑中, 与移动计算环境中其他服务的信息通信, 其数据集可为 $\emptyset$. 如果通信发生在相异节点, 其关联仅限于依据服务自身业务逻辑需要而产生的必要信息交换的输出结 果集.

- $D$ 表示定义 1 中表述的宿居设备.

- $\mathbf{P}_{a}$ 表示原子服务的可用性, 其受自身逻辑和各种上下文等因素影响而变化.

- $\mathbf{P}_{r}$ 表示原子服务的可靠性, 其受自身逻辑和各种上下文等因素影响而变化.

定义 3 (服务任务) 原子服务 $\mathrm{WS}_{a}$ 的任务 $I$ 可表示为一个六元组 $I=(P, T, F, \lambda, M, \mathrm{rfw})$, 其中:

- $P=P^{\mathrm{io}} \cup P^{s} \cup P^{c} \cup P^{\mathrm{qos}}, P^{\mathrm{io}}$ 描述任务 $I$ 的功能性指标, 即 $I$ 的输入/输出数据托肯 (Token) 集. $P^{s}$ 描述 $I$ 的状态托肯集 $P^{s} \subseteq\{$ Ready, Running, Activated, Committed, Aborted, Failed, Cancelled,

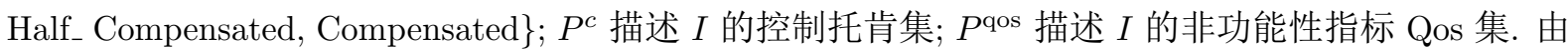
$I . P^{\mathrm{io}}, I . P^{s}, I . P^{c}$ 和 $I . P^{\mathrm{qos}}$ 获取 $I$ 的数据、状态、控制和非功能性参数, 其中 $I . P^{\mathrm{in}}$ 和 $I . P^{\mathrm{out}} \subseteq I . P^{\mathrm{io}}$.

- $T=T^{b} \cup T^{n} \cup \tau$, 其中 $T^{n}$ 描述 $I$ 上正向事件集 $\{\operatorname{Run}(), \operatorname{Activate}(), \operatorname{Abort}(), \operatorname{Fail}(), \operatorname{Commit}()$, $\operatorname{Cancel}()\}, T^{b}$ 描述 $I$ 上反向事件集 $\{\operatorname{HCompensate(),~Compensate(),~Retry()\} ,~} \tau \in T$ 为空事件. 其中 Activate () 激活任务, 记为 $t^{\text {act }} ; \operatorname{Run}()$ 执行任务, 记为 $t^{\mathrm{run}} ; \operatorname{Fail}()$ 执行任务失败, 记为 $t^{\mathrm{fal}} ; \operatorname{Abort}()$ 中 止任务执行, 记为 $t^{\mathrm{abt}} ; \operatorname{Cancel}()$ 取消执行, 记为 $t^{\mathrm{cnl}} ; \operatorname{Retry}()$ 重试失败执行, 记为 $t^{\mathrm{rty}} ; \operatorname{Commit}()$ 提交 任务执行, 记为 $t^{\mathrm{cmt}}$; Compensate() 补偿已完成任务, 记为 $t^{\mathrm{cmp}}$. 用 $I . t$ 获取 $I$ 的事件.

- $F=(P \times T) \cup(T \times P), P \times T$ 和 $T \times P$ 分别描述控制流和数据流, 正常执行序列 $\left\{\left(I . P^{\mathrm{in}}, I . t_{1}\right)\right.$, $\left.\left(I . t_{1}, I . p_{1}\right),\left(I . p_{1}, I . t_{2}\right),\left(I . t_{2}, I . p_{2}\right), \ldots,\left(I . p_{n-1}, I . t_{n}\right),\left(I . t_{n}, I . P^{\text {out }}\right)\right\}$.

- $\lambda=\left\{\lambda_{i} \mid \lambda_{i}=\lambda\left(t_{i}\right), 1 \leqslant i \leqslant n\right\}$, 其中 $\lambda_{i}$ 表示事件 $t_{i}$ 在规定时间 $\mathbf{t}$ 内点火延迟并成功转移的平均概 率, 即点火概率; $O\left(q_{i}\right)$ 表示事件 $t_{i}$ 在规定时间 $\mathbf{t}$ 内的点火关键观测值, 该值随上下文确定值类型 (如 时间和状态等), 简称点火观察值. 观测值满足指数分布 $\mathbf{P}\left(O\left(q_{i}\right)<x\right)=1-e^{-\lambda_{i} x}$, 其中 $q_{i}$ 是事件 $t_{i}$ 发 生的观测值, $x$ 是时间值, 期望值 $\mathrm{E}\left(O\left(q_{i}\right)\right)=\int_{x=0}^{\infty} \lambda_{i} e^{-\lambda_{i} x} x \mathrm{~d} x=1 / \lambda_{i}$. 任务间存在约束: $\left(t_{i}, p_{i}\right) \prec\left(t_{i+1}\right.$, $\left.p_{i+1}\right)$ 表示事件 $t_{i}$ 先于 $t_{i+1}$ 发生; $\left(t_{i}, p_{i}\right) \triangleleft \triangleright\left(t_{i+1}, p_{i+1}\right)$ 表示 $t_{i}$ 和 $t_{i+1}$ 相互排斥; $\left(t_{i}, p_{i}\right) \approx\left(t_{i+1}, p_{i+1}\right)$ 表示事件 $t_{i}$ 和 $t_{i+1}$ 同时发生或不发生. 它们存在如下基本的不确定性点火概率聚合:

(1) 若有任意 $t_{i}$ 和 $t_{i+1}$, 且同时发生, 有 $\mathbf{P}\left(O\left(q_{i}\right)<x\right)=1-e^{-\lambda_{i} x}$ 和 $\mathbf{P}\left(O\left(q_{i+1}\right)<(x+\Delta x)\right)=$ $1-e^{-\lambda_{i+1}(x+\Delta x)}$, 存在 $O\left(q_{i}\right)=O\left(q_{i+1}\right)$, 则 $t_{i}$ 与 $t_{i+1}$ 聚合事件概率 $\mathbf{P}\left(O\left(q_{i}\right)=O\left(q_{i+1}\right)\right)=\mathbf{P}\left(O\left(q_{i}\right)<\right.$ 
$x) * \mathbf{P}\left(x<O\left(q_{i+1}\right)<(x+\Delta x) \mid O\left(q_{i}\right)\right)$, 因为 $\lim _{\Delta x \rightarrow 0} \mathbf{P}\left(x<O\left(q_{i+1}\right)<(x+\Delta x) \mid O\left(q_{i}\right)\right)=1$, 也即 $\mathbf{P}\left(O\left(q_{i}\right)=O\left(q_{i+1}\right)\right)=\mathbf{P}\left(O\left(q_{i}\right)\right)$, 故两事件不必点火转移.

(2) 若 $\left(t_{i}, p_{i}\right) \prec\left(t_{i+1}, p_{i+1}\right)$, 则 $t_{i}$ 与 $t_{i+1}$ 聚合事件概率 $\mathbf{P}\left(O\left(q_{i}\right)<O\left(q_{i+1}\right)\right)=\mathbf{P}\left(O\left(q_{i}\right)<x\right) * \mathbf{P}(x<$ $\left.O\left(q_{i+1}\right)<(x+\Delta x) \mid O\left(q_{i}\right)\right)=\mathbf{P}\left(O\left(q_{i+1}\right)<(x+\Delta x)\right)$, 后可简记为 $\mathbf{P}\left(O\left(q_{i+1}\right)<x\right)$, 即两事件点火概率 聚合为后者的独立点火概率. 具体情况有:

- 托肯转移集 $M$, 即 $M_{i}(p) \stackrel{t}{\longrightarrow} M_{j}(p)$, 转移概率 $\mathbf{P}_{M i \rightarrow M j}=\mathbf{P}\left(M_{i} \stackrel{t_{i j}}{\longrightarrow} M_{j}\right) / \sum_{k} \mathbf{P}\left(M_{i} \stackrel{t_{i k}}{\longrightarrow} M_{j}\right)$. 由 $\mathbf{P}\left(O\left(q_{i}\right)>O\left(q_{j}\right)\right) /\left(\mathbf{P}\left(O\left(q_{i}\right)>O\left(q_{k}\right)\right)+\mathbf{P}\left(O\left(q_{k}\right)\right)>O\left(q_{j}\right)\right)=\lambda_{i} /\left(\lambda_{j}+\lambda_{k}\right)$, 那么 $\mathbf{P}_{M i \rightarrow M j} / \mathbf{P}_{M i \rightarrow M k}=$ $\lambda_{i j} / \lambda_{i k}$. 一般地 $\mathbf{P}_{M i \rightarrow M 1}: \mathbf{P}_{M i \rightarrow M 2}: \cdots: \mathbf{P}_{M i \rightarrow M k}=\lambda_{i 1}: \lambda_{i 2}: \cdots: \lambda_{i k}$, 则可标记 $M_{i} \rightarrow M_{j}$ 的转移概 率为 $\mathbf{P}_{M i j} /\left(\sum_{k} \mathbf{P}_{M i k}\right)=\lambda_{i j} /\left(\sum_{k} \lambda_{i k}\right)$. 对于任务的第 $i$ 阶段, 其托肯稳定分布概率 $\pi_{i}=\mathbf{P}\left(M_{i j}\right) /\left(\sum_{k}\right.$ $\left.\mathbf{P}\left(M_{i k}\right)\right)$, 且 $\sum_{i} \pi_{i}=1$, 任一稳定概率记 $\pi$.

- 应用资源需求映射到 $\mathrm{WS}_{a}$ 后的点火概率, 记为 $\mathrm{rfw}$, 若 $\mathrm{rfw} \in\{\pi, \chi\}$, 则点火成功, 且成功概率记为 $\lambda(\mathrm{rfw})$, 否则失败. 显然 $\chi$ 是 $\mathrm{rfw}$ 不在任务托肯稳定分布概率中的概率值. 那么映射概率 $\mathbf{P}_{r \rightarrow \mathrm{wsa}}(\mathrm{rfw})$ 存 在如下情形: (1) 若 $r f w=\chi$, 则 $\mathbf{P}_{r \rightarrow \mathrm{wsa}}(\mathrm{rfw})=0 ;(2)$ 若 $\mathrm{rfw}=\pi$, 则 $\mathbf{P}_{r \rightarrow \mathrm{wsa}}(\mathrm{rfw})=1 ;(3)$ 若 $\mathrm{rfw}=\mathrm{rfw}_{1} \oplus \mathrm{rfw}_{2}$, 则 $\mathbf{P}_{r \rightarrow w s a}(\mathrm{rfw})=\mathbf{P}_{r \rightarrow \mathrm{wsa}}\left(\mathrm{rfw}_{1}\right) * \mathbf{P}_{r \rightarrow \mathrm{wsa}}\left(\mathrm{rfw}_{2}\right) ;$ (4) 若 $\mathrm{rfw}=\mathrm{rfw}_{1} \mid \mathrm{rfw}_{2}$, 则 $\mathbf{P}_{r \rightarrow \mathrm{wsa}}(\mathrm{rfw})=\lambda\left(\mathrm{rfw}_{1}\right) \mathbf{P}_{r \rightarrow \mathrm{wsa}}$ $\left(\mathrm{rfw}_{1}\right)+\lambda\left(\mathrm{rfw}_{2}\right) \mathbf{P}_{r \rightarrow \mathrm{wsa}}\left(\mathrm{rfw}_{2}\right)$.

\section{3 移动环境下原子服务的事务机制}

移动原子服务 $\mathrm{WS}_{a}$ 具有独特的事务行为, 每个事务行为与其执行语义密切相关, 可分为: Pivot (枢 轴) 原子服务 $\mathrm{WS}_{a}^{p}$, Compensable (补偿) 原子服务 $\mathrm{WS}_{a}^{c}$, Retriable(重试) 原子服务 $\mathrm{WS}_{a}^{r}$ 和 Vital (关键) 原子服务 $\mathrm{WS}_{a}^{v}$, 其事务性质记为 $\mathrm{TBP}(\mathrm{WS}), \mathrm{WS}_{a}$ 提取事务属性记为 $\mathrm{WS}_{a} \cdot \mathrm{TBP}, \mathrm{WS}_{a} . \mathrm{TBP} \in\{\mathrm{Compen}-$ sable, Vital, Pivot, Retriable\}.

根据移动环境下事务执行过程, 任务 $I$ 可能处于 Activated, Ready, Committed, Running, Aborted, Failed, Cancelled, Half_Compensated, Compensated 等 9 个状态之一. 设任务 $I$ 的状态空间 $S$ 及其存 在概率 $\mathbf{P}_{I}^{s}$, 满足 $\sum_{s \in S} \mathbf{P}_{I}^{s}=1$. 在事务处理中 $\mathrm{WS}_{a}$ 可用性 $\mathbf{P}_{\mathrm{sa}}$ 可靠性 $\mathbf{P}_{\mathrm{sr}}$ 与执行状态相关, 如失败恢 复可增加整体可靠性, $\mathbf{P}_{\mathrm{sr}}\left(w s_{a}\right)$ 的值越大, $\mathrm{WS}_{a}$ 失效的概率越低, 可靠性越高. 为了更准确地分析基于 移动组合服务的事务情况，以及特定补偿事务如何影响其他的行为, 下面以基本场境讨论原子服务与 系统之间的行为依赖性.

实际上, 正是由于行为依赖性, 各状态中任务 $I$ 的可用性和可靠性通过流 $F$ 中传递. 在事务处理 机制中, 事务失效的判断依据可能是事务最终失败, 或者事务中某状态的可用性低于设定的阈值或可 靠性低于设定的阈值. 下述的事务讨论中将原子服务的可补偿性与可重试性视为两个正交属性, 其中, 任务 $I$ 界定为 $\mathrm{WS}_{a}$ 的动态形态, 可用性与可靠性将作为原子服务整体性能在下一章节介绍.

如图 1(a) 所示, $\mathrm{WS}_{a}^{p}$ 指服务既不是可补偿, 也不是可重试, $\mathrm{WS}_{a}$ 一旦执行成功, 语义上不能消除 其产生的影响. $\mathrm{WS}_{a}^{p}$ 的事件状态描述如下:

(1) 若按 $t_{1} t_{2}$ 执行, $I^{p} . P^{s}$ 为 Committed, 则其成功提交概率为 $\mathbf{P}_{I}^{\mathrm{cmt}}$, 如 $P_{0}, P_{1}$ 与 $P_{2}$ 可用性可靠 性良好, 可认为 $\mathbf{P}_{\mathrm{I}}=\mathbf{P}_{I}^{\mathrm{cmt}}=1$.

(2) 若按 $t_{1} t_{6}$ 执行, 那么 $I^{p} \cdot P^{s}$ 为 Failed, 则执行失效概率为 $\mathbf{P}_{I}^{f}$, 失效会降低可用性. $I^{p}$ 可用性 概率为 $\mathbf{P}_{I a}\left(I^{p}\right)=1-\mathbf{P}_{I}^{f}-\mathbf{P}_{\mathrm{Sys}}^{f}$, 且 $\mathbf{P}_{\mathrm{Sys}}^{f}$ 为其执行过程中的系统与环境失效概率.

(3) 若按 $t_{1} t_{3}$ 执行, 那么 $I^{p} . P^{s}$ 为 Aborted, 记中止概率为 $\mathbf{P}_{I}^{\text {abt }}$.

(4) 若按 $t_{1} t_{5}$ 执行, 那么 $I^{p} . P^{s}$ 为 Cancelled, 记取消概率为 $\mathbf{P}_{I}^{\mathrm{cnl}}$. 


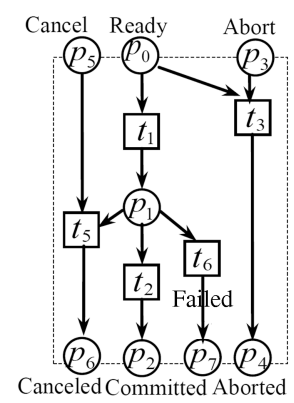

(a)

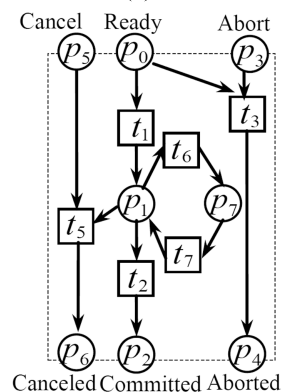

(c)

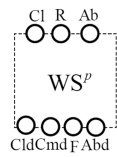

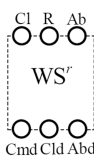

图 1 原子服务的状态转移图

(b)

(d)
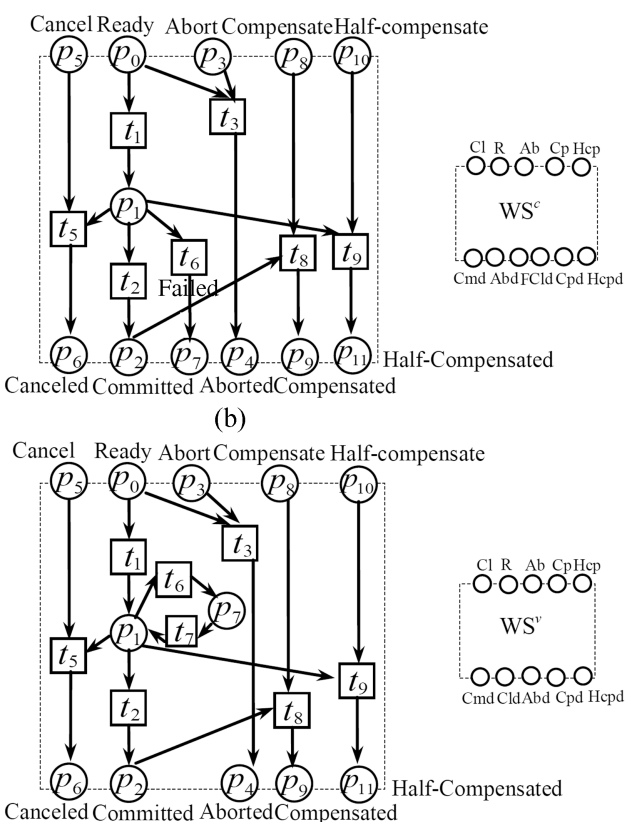

Figure 1 State transitions for typical atomic services. (a) Pivot service; (b) compensable service; (c) retriable service; (d) vital service

如图 1(b) 所示, 由于 $\mathrm{WS}_{a}^{p}$ 执行后不能消除已造成的影响. 我们考虑应用补偿服务 $\mathrm{WS}_{a}^{c}$, 它指原 子服务成功提交后, 执行其补偿服务消除所产生的影响, 其事务行为如下:

(1) 若按 $t_{1} t_{2} t_{8}$ 执行, $I^{c} . P^{s}$ 转到 Compensated, 则其补偿概率为 $\mathbf{P}_{I}^{\mathrm{cmp}}$.

(2) 若按 $t_{1} t_{3} t_{9}$ 执行, 取消 $I^{c}$ 并转到 Hcompensated, 则其部分补偿的概率为 $\mathbf{P}_{I}^{\mathrm{hcmp}}$.

由于 $\mathrm{WS}^{c}$ 包含 $I$ 和 $I^{\prime}$ (补偿任务), 执行 $I^{\prime}$ 消除 $I$ 产生的影响.

如图 1(c) 所示, $\mathrm{WS}_{a}^{r}$ 是重试服务, 指有限次执行 $\mathrm{WS}_{a}$ 后, 最终 $\mathrm{WS}_{a}$ 成功执行, 此况下 $\mathbf{P}_{I}^{\mathrm{cmt}}=1$. 由于 $\mathrm{WS}_{a}^{r}$ 不同于 $\mathrm{WS}_{a}^{p}$, 前者具有重试能力, 讨论行为如下:

若执行路径 $t_{1}\left(t_{6} t_{7}\right)^{n} t_{2}$ (其中 $n \geqslant 1, n$ 是重试次数), 有限次重试失败任务 $I^{r}, I^{r}$. $P^{s}$ 最终到达 Committed, 如 $P_{0}, P_{1}, P_{2}$ 与 $P_{7}$ 可用性可靠性良好, 可认为 $\mathbf{P}_{I}^{\mathrm{cmt}}=1$.

因此, $\mathrm{WS}_{a}^{r}$ 的任务 $I$, 若前 $k(k \leqslant n)$ 次均失败, 但经过 $k+1$ 至 $n$ 次 $I^{r} . P^{s}$ 最终到达 Committed, 则 $\mathbf{P}_{I}^{\mathrm{cmt}}=1$.

如图 1(d) 所示, $\mathrm{WS}_{a}^{v}$ 是指兼具可重试和可补偿能力, 其同时具有 $\mathrm{WS}_{a}^{c}$ 和 $\mathrm{WS}_{a}^{r}$ 事务处理能力.

若 $\mathrm{WS}^{\prime \prime}$ 是可替代 $\mathrm{WS}_{a}$, 那么存在相同功能语义, 那么 $I^{\prime \prime}$ 是 $I$ 的替代任务, 因此可扩展 $\mathrm{WS}_{a}$ 的 事务行为使其具可补偿、可重试或可替代恢复.

在实际执行原子任务 $I$ 时, 我们设计事务处理器 $(\mathrm{RH})$ 要获取任务的执行日志, 由 $I . p^{s}$ 执行状态 协调进展. 表 1 给出了状态和变迁含义.

\section{4 移动环境下原子服务的不确定性表征}

在上述原子服务模型中, 除了事务机制外, 移动环境带来的服务不确定性也是其重要关注属性之 
表 1 事务属性的变迁和库所

Table 1 Transitions and places in transaction model

\begin{tabular}{llllllll}
\hline Place & state & Place & State & Trans & Action & Trans & Action \\
\hline$p_{0}$ & Activated & $p_{6}$ & Cancelled & $t_{1}$ & Activate () & $t_{8}$ & Compensate () \\
$p_{1}$ & Running & $p_{7}$ & Failed & $t_{2}$ & Run () & $t_{9}$ & Half compensate () \\
$p_{2}$ & Committed & $p_{8}$ & Coordination compensated & $t_{3}$ & Abort () & & \\
$p_{3}$ & Coordination aborted & $p_{9}$ & Compensated & $t_{5}$ & Cancel () & & \\
$p_{4}$ & Aborted & $p_{10}$ & Coordination half compensated & $t_{6}$ & Fail () & & \\
$p_{5}$ & Coordination cancelled & $p_{11}$ & Half compensated & $t_{7}$ & Retry () & & \\
\hline
\end{tabular}

一, 为了在事务中实现有效处理, 必须完成服务不确定性表征. 由于移动计算中不确定性涉足内容十 分广泛, 本研究将其不确定性限指服务非功能方面的波动性, 具体仅含可用性 (availability) 和可靠性 (reliability) 方面的不确定性.

\section{1 移动环境下原子服务的可用性}

原子服务 $\mathrm{WS}_{a}$ 所处的非失败工作状态概率表示服务可用性. 鉴于移动计算环境的特殊性, 拟将 主要关注点限定于导致该环境中原子服务产生失败而不能正常工作的 3 类事件, 即能量故障、通信故 障和宿居环境下服务自身故障, 对 3 类故障分别建模, 最后按照三者的逻辑关系构建原子服务的可用 性. 能量问题是移动计算中服务的特性问题, 没有了电能的供给, 原子服务的宿居设备将停止工作, 服 务的正常工作状态亦不复存在; 在原子服务存在涉外数据交换的情况下, 通信链路硬故障的出现将同 样会损毁原子服务的既定执行, 使其处于故障工作状态; 在既有的宿居环境下, 当工作负载超出宿居 节点设备的工作强度极限时, 原子服务的自身工作环境会发生质的恶化, 即出现 “系统崩溃”, 此时, 原 子服务也将不可能正常工作, 即处于另一种失效状态. 为此, 本文将对三者分别进行分析.

\subsection{1 $\mathrm{WS}_{a}$ 能量 $P_{\mathrm{ow}}$ 的可用性}

设原子服务宿居设备 $D$ 的平均工作周期为 $\mathbf{T}_{\mathrm{cyc}}$ ，指处于移动计算环境中服务宿居节点设备从 自身能量资源处于最好状态 (一般指电能的充满状态), 到其资源由于服务工作的消耗而逐渐衰减, 直 至应用殆尽而处于能量故障状态的平均时间长度. 显然, 它是宿居节点设备在不同无线网络下的应用 经验值, 从一般实际工作情况来看, 它关联有效电能工作时间 $\mathbf{t}_{\mathrm{Pow}}$ 和电能的耗尽待充 (或替换) 时间 $\mathbf{t}_{\text {down }}$. 项目将时间限定于 $\mathbf{T}_{\mathrm{cyc}}$ 周期内进行建模, 认为正常时间 $\mathbf{t}_{\mathrm{po}}$ 是此周期的简单重复, 即便于研究 讨论的需要, 限定于 $\mathbf{t}_{\mathrm{po}} \leqslant \mathbf{T}_{\mathrm{cyc}}$.

按照假设 (9), 设原子服务宿居节点设备的总电能为 $\left|P_{o w}\right|$, 电能消耗量强度为 $\xi_{\mathrm{Pow}}$, 在节点设备 工作时间 $\mathbf{t}_{\mathrm{po}}$ 内, 如果 $0 \leqslant \mathbf{t}_{\mathrm{po}} \leqslant \mathbf{t}_{\mathrm{Pow}} \wedge\left|P_{\mathrm{ow}}\right|=\xi_{\mathrm{Pow}} \mathbf{t}_{\mathrm{Pow}} \wedge \mathbf{t}_{\mathrm{cyc}}=\mathbf{t}_{\mathrm{Pow}}+\mathbf{t}_{\mathrm{down}}$, 那么电能消耗量 $P_{\text {o_used }}=\xi_{\text {Pow }} \mathbf{t}_{\mathrm{po}}$; 如果 $\mathbf{t}_{\mathrm{Pow}}<\mathbf{t}_{\mathrm{po}} \leqslant \mathbf{t}_{\mathrm{cyc}} \wedge\left|P_{\mathrm{ow}}\right|=\xi_{\text {Pow }} \mathbf{t}_{\mathrm{Pow}} \wedge \mathbf{t}_{\mathrm{cyc}}=\mathbf{t}_{\mathrm{Pow}}+\mathbf{t}_{\mathrm{down}}$, 那么 $P_{\text {o_used }}=\left|P_{\mathrm{ow}}\right|$.

设 $\mathbf{P}_{\mathrm{sa}}\left(P_{\mathrm{ow}}\right)$ 表示 $P_{\mathrm{ow}}$ 的可用概率, 基于 $P_{\mathrm{ow}}$ 的可用状况表述, 为了计算 $P_{\mathrm{ow}}$ 可用概率的便利, 不失一般性, 对于具体原子服务而言, 可以假定在一个应用周期 $\mathbf{t}_{\mathrm{cyc}}$ 中 $\mathbf{t}_{\mathrm{po}}$ 服从有界对数正态分布 (由于 $0 \leqslant \mathbf{t}_{\mathrm{po}} \leqslant \mathbf{t}_{\mathrm{cyc}}$, 其上界变换式为 $\mathbf{t}_{\mathrm{po}} /\left(\mathbf{t}_{\mathrm{cyc}}-\mathbf{t}_{\mathrm{po}}\right)$, 由历史统计可获得变换式的平均值为 $\mu_{t}$, 变换式的标准差为 $\sigma_{t}$, 则 $\mathbf{t}_{\mathrm{po}}$ 概率密度函数为 $\mathrm{f}_{\mathbf{t p o}}\left(\mathbf{t}_{\mathrm{po}}, \mu_{t}, \sigma_{t}\right)=\left(\mathbf{t}_{\mathrm{cyc}}-\mathbf{t}_{\mathrm{po}}\right) \exp \left(-\left(\ln \left(\mathbf{t}_{\mathrm{po}} /\left(\mathbf{t}_{\mathrm{cyc}}-\mathbf{t}_{\mathrm{po}}\right)\right)\right.\right.$ $\left.\left.-\mu_{t}\right)^{2} / 2 \sigma_{t}^{2}\right) /\left(\mathbf{t}_{\mathrm{po}} \sigma_{t} \sqrt{2 \pi}\right)$. 
由 $P_{\mathrm{o} \_u s e d}$ 和 $\mathrm{f}_{\mathbf{t p o}}\left(\mathbf{t}_{\mathrm{po}}, \mu_{t}, \sigma_{t}\right)$ 知 $P_{\mathrm{ow}}$ 的可用状态由 $\mathbf{t}_{\mathrm{po}}$ 确定, 并对 $\mathbf{t}_{\mathrm{po}}$ 直接积分可得到的 $P_{\mathrm{ow}}$ 可 用性, 因此, 可用概率:

$$
\begin{aligned}
\mathbf{P}_{\mathrm{sa}}\left(P_{\mathrm{ow}}\right) & =\int_{0}^{\mathbf{t}_{\mathrm{pow}}} f_{x}\left(x, \mu_{t}, \sigma_{t}\right) \mathrm{d} x \\
& =\int_{0}^{\mathbf{t}_{\mathrm{pow}}}\left(\left(\mathbf{t}_{\mathrm{cyc}}-x\right) \exp \left(-\left(\ln \left(x /\left(\mathbf{t}_{\mathrm{cyc}}-x\right)\right)-\mu_{t}\right)^{2} / 2 \sigma_{t}^{2}\right) /\left(x \sigma_{t} \sqrt{2 \pi}\right)\right) \mathrm{d} x .
\end{aligned}
$$

\subsection{2 $\mathrm{WS}_{a}$ 通信链路及其 $C$ 的可用性}

设移动计算环境中无线网络的无线发射功率和频率恒定, 且原子服务宿居节点设备的接受天线增 益恒定, 并假定信号传播介质为自由空间. 设原子服务宿居节点设备的无线信号接受灵敏度为 $\operatorname{Sin}_{c}$ (单 位 $\mathrm{dBm}$, 在 $802.11 \mathrm{~b} / \mathrm{g}$ 中一般要求在 $-80 \mathrm{dBm}$ 左右 $)$, 无线网络的发射信号总量为 $\operatorname{Ts}_{c}($ 单位 $\mathrm{dBm}$, 在 $802.11 \mathrm{~b} / \mathrm{g}$ 环境中目前普通室内 $\mathrm{AP}$ 的发射及其增益量为 $25 \mathrm{dBm}$ 左右). 由于通信传输损耗 $\operatorname{Los}_{c}$, 通 信距离 $d_{c}$ 和工作频率 $f_{c}$ 满足 $\operatorname{Los} c=A_{0}+20 \log \left(d_{c}\right)+20 \log \left(f_{c}\right)$, 其中常量 $A_{0}$ 一般取值为 32.44 .

由于当原子服务宿居节点设备的接受信号量 $\mathrm{Rs}_{c}$ (即 $\mathrm{Ts}_{c}-\operatorname{Los}_{c}$ ) 低于接受灵敏度 $\operatorname{Sin}_{c}$ 时, 原子服 务的 $C$ 将处于故障状态, 不可能完成对外节点的数据交换, 则认为 $C$ 处于不可用状态. 设原子服务在 对外进行数据通信期间, 通信链路的可用状况保持一致. 由于 $C$ 的可用状况藉由原子服务节点设备所 处通信链路与对外通信量 $|C|$ 所决定, 一般而言, $|C|=0$ 意味单个原子服务完成的业务逻辑就是用户 最终需要的全部应用逻辑, 显然在传统网络环境中来自这方面的影响较小, 但在移动计算中由于服务 外部上下文的 “恶化” (如无线网络的不确定性增强), 这种单原子服务完成的应用的比例不容忽视, 设 其概率为 $\mathbf{P}_{C}$ (采用历史经验值), 即 $|C|=0$ 的概率为 $\mathbf{P}_{C 0}$.

设原子服务宿居节点设备与无线信号发射点 (即中心节点设备) 的距离也为 $d_{c}$, 不失一般性, 因 为 $d_{c} \geqslant 0$ 则可以认为 $d_{c}$ 服从对数正态分布, 由历史经验值可获得 $d_{c}$ 的对数的平均值为 $\mu_{d}, d_{c}$ 的对 数的标准差为 $\sigma_{d}$, 则其概率密度 $f_{d}\left(d_{c}, \mu_{d}, \sigma_{d}\right)=\exp \left(-\left(\ln \left(d_{c}\right)-\mu_{d}\right)^{2} /\left(2 \sigma_{d}^{2}\right)\right) /\left(d_{c} \sigma_{d} \sqrt{2 \pi}\right)$. 设原子服务宿 居节点设备能正常接收到无线网络信号的临界距离为 $\operatorname{dis}(C)=\sqrt[20]{10^{\left(T x_{c}-R s_{c}-A_{0}\right)}} / f_{c}$, 可以计算得到 通信链路的可用概率 $\mathbf{P}_{\mathrm{sa}}(L C)=\int_{0}^{\operatorname{dis}(C)} f_{x}\left(x, \mu_{d}, \sigma_{d}\right)$.

由于 $|C|=0$ (设该情况存在概率为 $\mathbf{P}_{C 0}$ ) 时, 通信链路对原子服务的可用性不产生影响, 仅在 $|C| \neq 0$ (概率为 $1-\mathbf{P}_{C 0}$ ) 时, $\mathbf{P}_{\mathrm{sa}}(C)$ 才通过 $C$ 作用于原子服务. 由前述假设知原子服务在对外通信期间, 通 信链路可用概率不变, 可得 $C$ 的可用概率

$$
\mathbf{P}_{\mathrm{sa}}(C)=\mathbf{P}_{C 0}+\left(1-\mathbf{P}_{C 0}\right) \int_{0}^{\sqrt[20]{10^{\left(T x_{c}-R s_{c}-A_{0}\right)}} / f_{c}}\left(\exp \left(-\left(\ln (x)-\mu_{d}\right)^{2} /\left(2 \sigma_{d}^{2}\right)\right) /\left(x \sigma_{d} \sqrt{2 \pi}\right)\right) \mathrm{d} x .
$$

\subsection{3 $\mathrm{WS}_{a}$ 软件体 $E$ 的可用性}

由前述概念模型及相关假设, 可知原子服务软件体 $E$ 的可用性与下述两方面因素决定, 即软体 自身工作强度 (如 $C_{\mathrm{cpu}}$ 和 $M_{\mathrm{in}}$ 等应用强度), 其宿居节点设备的工作负载. 同时宿居于同一节点设备 的各原子服务软体的总工作强度是有限的, 对于服务宿居节点设备 $D$ 的 $C_{\mathrm{cpu}}$ 而言, 极限工作强度为 $\operatorname{Max}_{c}$, 而对应 $M_{\mathrm{in}}$ 为 $\mathrm{Max}_{m}$, 它们可按照设备历史经验值获取. 令某 $\mathrm{WS}_{a}$ 在宿居节点的 $C_{\mathrm{cpu}}$ 的工作 强度为 $G_{\mathrm{cpu}}$, 在 $M_{\mathrm{in}}$ 上的工作强度为 $G_{\mathrm{mem}}$, 按照假设 $(4),(8),(10)$ 和 (11), 同时可假定同一节点设备 的各 $\mathrm{WS}_{a}$ 工作强度情况一样. 因为原子服务工作强度为一个确定值的发生事件是随机独立事件, 且 $G_{\mathrm{cpu}}$ 和 $G_{\mathrm{mem}}$ 皆为非负实数, 两类事件都遵从指数正态分布. 
对于节点工作强度 $G_{\mathrm{cpu}}$ 的指数正态分布而言, 由历史经验值可获得 $C_{\mathrm{cpu}}$ 工作强度的平均值为 $\bar{G}_{\mathrm{cpu}}$, 其对数的平均值为 $\mu_{G c}, C_{\mathrm{cpu}}$ 工作强度的对数的标准差为 $\sigma_{G c}$, 则 $G_{\mathrm{cpu}}$ 概率密度 $\mathrm{f}_{\mathrm{Gcpu}}\left(G_{\mathrm{cpu}}\right.$, $\left.\mu_{G c}, \sigma_{G c}\right)=\exp \left(-\left(\ln \left(G_{\mathrm{cpu}}\right)-\mu_{G c}\right)^{2} /\left(2 \sigma_{G c}^{2}\right)\right) /\left(G_{\mathrm{cpu}} \sigma_{G c} \sqrt{2 \pi}\right)$.

设 $N_{D}$ 表示节点设备某时刻的宿居 $\mathrm{WS}_{a}$ 的总个数, 不失一般性, 设 $\omega$ 为节点设备单位时间 $\mathrm{WS}_{a}$ 期望到达数, 则随机变量 $N_{D}$ 服从参数为 $\omega(\omega>0)$ 的泊松分布, 分布概率 $\mathbf{P}\left(N_{D}=n\right)=\exp (-\omega) \omega^{n} / n !$, 其中 $n=0,1,2, \ldots, N$. 根据前述假设, 当 $N_{D} \leqslant \mathrm{Max}_{c} / G_{\mathrm{cpu}}$ 时, $\mathrm{WS}_{a}$ 基于 $C_{\mathrm{cpu}}$ 皆可用. 由于 $C_{\mathrm{cpu}}$ 的 平均工作强度为 $\bar{G}_{\mathrm{cpu}}$, 在 $\mathrm{WS}_{a}$ 宿居节点在 $N_{D} \leqslant \mathrm{Max}_{c} /\left(\bar{G}_{\mathrm{cpu}}\right)$ 情况下, 皆不会产生 “崩溃” 失败, $\mathrm{WS}_{a}$ 基于 $C_{\mathrm{cpu}}$ 的可用概率 $\mathbf{P}_{\mathrm{sa}}\left(G_{\mathrm{cpu}}\right)$, 即为 $N_{D} \leqslant \mathrm{Max}_{c} /\left(\bar{G}_{\mathrm{cpu}}\right)$ 下 $\mathrm{WS}_{a}$ 分布的概率之和 $\mathbf{P}_{\mathrm{sa}}\left(G_{\mathrm{cpu}}\right)=$ $\Sigma_{n=1}^{\mathrm{Max}_{c} / \bar{G}_{\mathrm{cpu}}}\left(\exp (-\omega) \omega^{n} / n !\right)$.

类似上述分析, 对于节点 $M_{\mathrm{in}}$ 工作强度 $G_{\mathrm{mem}}$ 的指数正态分布而言, 设获得的 $M_{\mathrm{in}}$ 平均工作强 度为 $\bar{G}_{\mathrm{mem}}$, 其对数的平均值为 $\mu_{G m}$, 对数的标准差为 $\sigma_{G m}, G_{\mathrm{mem}}$ 概率密度函数 $f_{\mathrm{Gmem}}\left(G_{\mathrm{mem}}, \mu_{G m}\right.$, $\left.\sigma_{G m}\right)=\exp \left(-\left(\ln \left(G_{\mathrm{mem}}\right)-\mu_{G m}\right)^{2} /\left(2 \sigma_{G m}^{2}\right)\right) /\left(G_{\mathrm{mem}} \sigma_{G m} \sqrt{2 \pi}\right)$. 当 $N_{D} \leqslant \operatorname{Max}_{m} / G_{\mathrm{mem}}$ 时, $\mathrm{WS}_{a}$ 基于 $M_{\mathrm{in}}$ 皆可用.

同样, 原子服务基于 $M_{\mathrm{in}}$ 的可用概率 $\mathbf{P}_{\mathrm{sa}}\left(G_{\mathrm{mem}}\right)$ 即为 $N_{D} \leqslant \mathrm{Max}_{m} /\left(\bar{G}_{\mathrm{mem}}\right)$ 下 $\mathrm{WS}_{a}$ 分布的概率 之和 $\left.\mathbf{P}_{s a}\left(G_{\mathrm{mem}}\right)=\Sigma_{n=1}^{\mathrm{Max}_{m} / \bar{G}_{\mathrm{mem}}}\left(\exp (-\omega) \omega^{n} / n\right) !\right)$. 按照假设, $\mathrm{WS}_{a}$ 软件体 $E$ 的可用性仅与 $C_{\mathrm{cpu}}, M_{\mathrm{in}}$ 和 $N_{D}$ (指代宿居节点设备的工作负载) 并列相关. 由此, 如果 $N_{D} G_{\mathrm{cpu}} \leqslant \operatorname{Max}_{c} \wedge N_{D} G_{\mathrm{mem}} \leqslant \mathrm{Max}_{m}, \mathrm{WS}_{a}$ 软件体 $E$ 的可用状态可用.

综上, 推导出 $\mathrm{WS}_{a}$ 软件体 $E$ 的可用概率:

$$
\mathbf{P}_{\mathrm{sa}}(E)=\mathbf{P}_{\mathrm{sa}}\left(G_{\mathrm{cpu}}\right) \cdot \mathbf{P}_{\mathrm{sa}}\left(G_{\mathrm{mem}}\right)=\prod_{x=\operatorname{Max}_{c} / \bar{G}_{\mathrm{cpu}}}^{\mathrm{Max}_{m} / \bar{G}_{\mathrm{mem}}} \Sigma_{n=1}^{x}\left(\exp (-\omega) \omega^{n} / n !\right) .
$$

设原子服务 $\mathrm{WS}_{a}$ 的整体可用概率 $\mathbf{P}_{\mathrm{sa}}\left(\mathrm{WS}_{a}\right)$, 同样基于 $\mathbf{P}_{\mathrm{sa}}\left(P_{\mathrm{ow}}\right), \mathbf{P}_{\mathrm{sa}}(C)$ 和 $\mathbf{P}_{\mathrm{sa}}(E)$, 可获得 $\mathbf{P}_{\mathrm{sa}}\left(\mathrm{WS}_{a}\right)=\mathbf{P}_{\mathrm{sa}}\left(P_{\mathrm{ow}}\right) \cdot \mathbf{P}_{\mathrm{sa}}(C) \cdot \mathbf{P}_{\mathrm{sa}}(E)$, 有:

$$
\begin{aligned}
\mathbf{P}_{\mathrm{sa}}\left(\mathrm{WS}_{a}\right)= & \prod_{x=\mathrm{Max}_{c} / \bar{G}_{\mathrm{cpu}}}^{\mathrm{Max}_{m} / \bar{G}_{\mathrm{mem}}} \sum_{n=1}^{x}\left(\exp (-\omega) \omega^{n} / n !\right) \int_{0}^{\mathbf{t}_{\mathrm{pow}}}\left(( \mathbf { t } _ { \mathrm { cyc } } - y ) \operatorname { e x p } \left(-\left(\ln \left(y /\left(\mathbf{t}_{\mathrm{cyc}}-y\right)\right)\right.\right.\right. \\
& \left.\left.\left.-\mu_{t}\right)^{2} / 2 \sigma_{t}^{2}\right) /\left(y \sigma_{t} \sqrt{2 \pi}\right)\right) \mathrm{d} y\left(\mathbf{P}_{C 0}+\left(1-\mathbf{P}_{C 0}\right) \int_{0}^{\sqrt[20]{10^{\left(T x_{c}-R s_{c}-A_{0}\right)} / f_{c}}}(\exp (-(\ln (z)\right. \\
& \left.\left.\left.\left.-\mu_{d}\right)^{2} /\left(2 \sigma_{d}^{2}\right)\right) /\left(z \sigma_{d} \sqrt{2 \pi}\right)\right) \mathrm{d} z\right) .
\end{aligned}
$$

\section{2 移动环境下原子服务的可靠性}

原子服务 $\mathrm{WS}_{a}$ 在移动计算环境中不产生失效且能正确执行的属性, 即 $\mathrm{WS}_{a}$ 的可靠性, 它由 $\mathrm{WS}_{a}$ 宿居硬件可靠性, 自身软体可靠性以及涉外通信链路的可靠性共同决定. 下面分别对其可靠性进行计 算, 然后形成为整体 $\mathrm{WS}_{a}$ 可靠模型.

\subsection{1 $\mathrm{WS}_{a}$ 的 $C_{\mathrm{cpu}}$ 的可靠性}

设 $\rho_{c}$ 为 $\mathrm{WS}_{a}$ 的计算复杂度, $\eta_{c}$ 为 $\mathrm{WS}_{a}$ 所宿居节点设备 $D$ 的运算速度, 那么原子服务在节点 $D$ 上 的运行所需时间 $\mathbf{t}_{\rho}=\rho_{c} / \eta_{c}$. 根据假设 (4), 设 $\omega$ 为单位时间原子服务期望到达数, $\mathbf{P}\left(\mathbf{t}_{\rho}, N_{D}=n\right)$ 指在 时间 $\mathbf{t}_{\rho}$ 内节点设备 $D$ 上同时宿居 $n$ 个原子服务的概率, $\mathbf{P}\left(\mathbf{t}_{\rho}, N_{D}=n\right)=\left(\omega \cdot \rho_{c} / \eta_{c}\right)^{n} \exp \left(-\omega \cdot \rho_{c} / \eta_{c}\right) / n !$, 其中 $n=0,1,2, \ldots, N$. 
令 $\operatorname{Int}\left(C_{\mathrm{cpu}}, n\right)$ 表示节点设备 $D$ 在 $n$ 个独立 $\mathrm{WS}_{a}$ 宿居下的 $\mathrm{CPU}$ 失效强度, 记 $\mathrm{Int}_{c}$, 并根据假设 (6) 和假设 (8) 可知, 设备 $D$ 上有 $n$ 个服务在执行时的同一时间内服务序列 $\mathrm{WS}_{a i}(i=0,1,2, \ldots, n)$ 为 独立同指数分布的随机变量. 显然, $\operatorname{Int}_{c}=f_{\mathrm{cpu}}\left(\mathrm{cpu}, n, \mathbf{t}_{\rho}\right)$, 其中 $f_{\mathrm{cpu}}$ 表示与设备个体 $D$ (主要是 $\mathrm{CPU}$ 计算力) 及宿居服务数 $n$, 时间 $\mathbf{t}_{\rho}$ 满足一定经验函数关系. $C_{\mathrm{cpu}}$ 不发生失效的概率 (即其可靠概率) 为

$$
\mathbf{P}_{\mathrm{sr}}(\mathrm{cpu})=\sum_{n=1}^{N}\left(\exp \left(-\operatorname{Int}_{c} \cdot \mathbf{t}_{\rho}\right) \cdot \mathbf{P}\left(\mathbf{t}_{\rho}, N_{D}=n\right)\right)=\sum_{n=1}^{N}\left(\left(\omega \cdot \rho_{c} / \eta_{c}\right)^{n} \exp \left(\left(-\omega-\operatorname{Int}_{c}\right) \cdot \rho_{c} / \eta_{c}\right) / n !\right) .
$$

\subsection{2 $\mathrm{WS}_{a}$ 的 $\mathrm{M}_{\mathrm{in}}$ 的可靠性}

令 $\operatorname{Int}\left(M_{i n}, n\right)$ 表示节点设备 $\mathrm{D}$ 在 $n(=0,1,2, \ldots, N)$ 个独立原子服务宿居下的 $M_{\mathrm{in}}$ 失效强度, 记 $\mathrm{Int}_{m}$, 同样根据假设 (6) 和假设 (8) 可知, 设备 $D$ 上有 $n$ 个服务在执行时的同一时间内服务序列 $w s_{\mathrm{ai}}(i=0,1,2, \ldots, n)$ 为独立同指数分布的随机变量. 显然, $\operatorname{Int}_{m}=f_{m}\left(m_{\mathrm{in}}, n, \mathbf{t}_{\rho}\right)$, 其中 $f_{m}$ 表示与其 个体 $D$ (主要是内存容量与属性), 宿居服务数 $n$ 和时间 $\mathbf{t}_{\rho}$ 满足一定经验函数关系. $M_{\mathrm{in}}$ 的可靠概率为

$$
\mathbf{P}_{\mathrm{sr}}\left(M_{\mathrm{in}}\right)=\sum_{n=1}^{N}\left(\exp \left(-\operatorname{Int}_{m} \cdot \mathbf{t}_{\rho}\right) \cdot \mathbf{P}\left(\mathbf{t}_{\rho}, N_{D}=n\right)\right)=\sum_{n=1}^{N}\left(\left(\omega \cdot \rho_{c} / \eta_{c}\right)^{n} \exp \left(\left(-\omega-\operatorname{Int}_{m}\right) \cdot \rho_{c} / \eta_{c}\right) / n !\right) .
$$

\subsection{3 $\mathrm{WS}_{a}$ 的 $C$ 的可靠性}

假设 $\mathrm{WS}_{a}$ 宿居节点设备 $D$ 在与外节点进行数据交换时，总是经由中心节点设备 (如 AP 设备) 完成; 中心节点是有线网络连接设备, 假定中心节点与外节点 (因为移动环境中节点设备 $D$ 需要与 其通信) 之间的传输可靠且平均速度为 $\eta_{0}$. 原子服务的数据交换传输量为 $|C|$. 服务经由宿居设备 $D$ 与中心节点之间通信链路的平均传输速度为 $\eta_{d c}$. 借助普适计算中的位置感知服务, 可以得到宿居 设备 $D$ 和中心节点的空间坐标分别 $\left(x_{d}, y_{d}, z_{d}\right)$ 和 $\left(x_{0}, y_{0}, z_{0}\right)$, 宿居设备 $D$ 与中心节点之间距离为 $d_{c c}=\sqrt{\left(x_{d}-x_{0}\right)^{2}+\left(y_{d}-y_{0}\right)^{2}+\left(z_{d}-z_{0}\right)^{2}}$.

无线传输情况拟以广泛应用的 IEEE $802.11 \mathrm{WLAN}$ 为例予以确定. 无线网络中因为传输距离的 增长而加速传播损耗, 为此网络将自适应调整数据传输速率 (如: $802.11 \mathrm{~g}$ 自适应 $1 \mathrm{Mb} / \mathrm{s} \sim 54 \mathrm{Mb} / \mathrm{s}$ 中 的 12 个传输速率). 设 $\max$ 为无线网络传输的最高速度级别数 (在 $802.11 \mathrm{~g}$ 中 $\max =12$ ), 因此, 不 失一般性, 如果 $0 \leqslant d_{\mathrm{cc}}<d_{\mathrm{cc} 1}$, 则 $\eta_{\mathrm{dc}}=\eta_{\max }$; 如果 $d_{\mathrm{cc} 1} \leqslant d_{\mathrm{cc}}<d_{\mathrm{cc} 2}$, 则 $\eta_{d c}=\eta_{\max -1} \ldots$, 如果 $d_{\mathrm{ccmax}-1} \leqslant d_{\mathrm{cc}}<d_{\mathrm{cccmax}}$, 则 $\eta_{d c}=\eta_{1}$. 因此 $\mathrm{WS}_{a}$ 完成 $|C|$ 通信需要的传输时间 $\mathbf{t}_{c}$ 也为分段变量, $\mathbf{t}_{c}=|C| / \eta_{i}+|C| / \eta_{0}$, 例如 $0 \leqslant d_{\mathrm{cc}}<d_{\mathrm{cc} 1}$ 时, $\eta_{i}=\eta_{\text {max }}$.

根据建模需要, 可设宿居设备 $D$ 与中心节点之间距离 $d_{\mathrm{cc}}$ 遵从 $f_{d c}\left(\mathbf{t}_{c}, \mu_{d c}, \sigma_{d c}\right)$ 表征的指数正态 分布, 其中 $\mu_{d c}$ 对数的平均值, $\sigma_{d c}$ 为标准差. 根据实际情况, $d_{\mathrm{cc}}$ 存在可靠通信的极限距离 $\operatorname{dis}(C)$, 超出 此距离则 $\mathrm{WS}_{a}$ 处于不可用状态 (已在可用模型中表达). 为了直接应用 $f_{d c}\left(\mathbf{t}_{c}, \mu_{d c}, \sigma_{d c}\right.$ ), 计算可靠传输 时间 $\mathbf{t}_{c}$ 的平均值 $\overline{\mathbf{t}_{c}}$ 如下: 首先计算归一化因子 $\psi_{d c}=\int_{0}^{+\infty} f_{d c}\left(x, \mu_{d c}, \sigma_{d c}\right) \mathrm{d} x / \int_{0}^{\operatorname{dis}(c)} f_{d c}\left(x, \mu_{d c}, \sigma_{d c}\right) \mathrm{d} x$, 即 $1 / \int_{0}^{\operatorname{dis}(c)}\left(\exp \left(-\left(\ln (x)-\mu_{d c}\right)^{2} /\left(2 \sigma_{d c}^{2}\right)\right) /\left(x \sigma_{d c} \sqrt{2 \pi}\right)\right) \mathrm{d} x$, 使其该指数正态分布中极限距离 $\operatorname{dis}(C)$ 以外 情况不在可靠模型考虑之列, 然后得 $\overline{\mathbf{t}_{c}}=|C| / \eta_{0}+\psi_{d c} \Sigma_{i=1}^{\max }\left(|C| \int_{\mathrm{dccmax}-i}^{\mathrm{dccmax}-i+1} f_{d c}\left(x, \mu_{d c}, \sigma_{d c}\right) \mathrm{d} x / \eta_{i}\right)$, 即为 $|C| / \eta_{0}+\psi_{d c} \Sigma_{i=1}^{\max }\left(|C| \int_{\operatorname{dccmax}-i}^{\operatorname{dccmax}-i+1}\left(\exp \left(-\left(\ln (x)-\mu_{d c}\right)^{2} /\left(2 \sigma_{d c}^{2}\right)\right) /\left(x \sigma_{d c} \sqrt{2 \pi}\right)\right) \mathrm{d} x / \eta_{i}\right)$.

根据假设 (5), 令 $\operatorname{Int}\left(\mathrm{LC}_{\mathrm{cpu}}, n\right)$ 表示原子服务宿居设备节点与对外通信节点间的通信链路的失效 强度, 记 $\mathrm{Int}_{L}$. 在通信数据集 $C$ 上, 该通信链路的可靠概率 (不失效, 其中 $\mathbf{t}_{c}$ 取 $\overline{\mathbf{t}_{c}}$ ) 为 


$$
\begin{aligned}
& \mathbf{P}_{\mathrm{sr}}(C)=\exp \left(-\operatorname{Int}_{L} \cdot \mathbf{t}_{c}\right)=\exp \left(-\operatorname{Int}_{L} \cdot \mathrm{A}\right), \text { 其中 } \\
& \quad A=|C| / \eta_{0}+\psi_{d c} \sum_{i=1}^{\max }\left(|C| \int_{\mathrm{dccmax}-i}^{\mathrm{dccmax}-i+1}\left(\exp \left(-\left(\ln (x)-\mu_{d c}\right)^{2} /\left(2 \sigma_{d c}^{2}\right)\right) /\left(x \sigma_{d c} \sqrt{2 \pi}\right)\right) \mathrm{d} x / \eta_{i}\right) .
\end{aligned}
$$

\subsection{4 $\mathrm{WS}_{a}$ 软件体 $E$ 的可靠性}

根据假设 (8), 令 $\operatorname{Int}(E, n)$ 表示原子服务构成软件体的失效强度, 记 $\operatorname{Int}_{E}$, 显然其观察时间 $\mathbf{t}_{o}=$ $\mathbf{t}_{\rho}+\mathbf{t}_{c}$, 则该构成软件体不发生失效的概率计算为 $\mathbf{P}_{\mathrm{sr}}(E)=\exp \left(-\operatorname{Int}_{E} \cdot\left(\mathbf{t}_{\rho}+\mathbf{t}_{c}\right)\right)$.

$\mathbf{P}_{\mathrm{sr}}(E)=\exp \left(-\operatorname{Int}_{E}\left(\rho_{c} / \eta_{c}+A\right)\right)$. 其中:

$$
A=|C| / \eta_{0}+\psi_{d c} \sum_{i=1}^{\max }\left(|C| \int_{\operatorname{dccmax}-i}^{\mathrm{dccmax}-i+1}\left(\exp \left(-\left(\ln (x)-\mu_{d c}\right)^{2} /\left(2 \sigma_{d c}^{2}\right)\right) /\left(x \sigma_{d c} \sqrt{2 \pi}\right)\right) \mathrm{d} x / \eta_{i}\right) .
$$

设原子服务 $\mathrm{WS}_{a}$ 的整体可靠概率为 $\mathbf{P}_{\mathrm{sr}}\left(\mathrm{WS}_{a}\right)$, 根据假设 (8) 并基于 $\mathbf{P}_{\mathrm{sr}}(\mathrm{cpu}), \mathbf{P}_{\mathrm{sr}}(\mathrm{mem}), \mathbf{P}_{\mathrm{sr}}(C)$ 和 $\mathbf{P}_{\mathrm{sr}}(E)$, 可获得 $\mathbf{P}_{\mathrm{sr}}\left(\mathrm{WS}_{a}\right)$ 为

$$
\begin{aligned}
\mathbf{P}_{\mathrm{sr}}\left(\mathrm{WS}_{a}\right) & =\mathbf{P}_{\mathrm{sr}}(\mathrm{cpu}) \cdot \mathbf{P}_{\mathrm{sr}}(\mathrm{mem}) \cdot \mathbf{P}_{\mathrm{sr}}(C) \mathbf{P}_{\mathrm{sr}}(E) \\
& =\prod_{g=\mathrm{cpu}}^{\operatorname{mem}} \sum_{n=1}^{N}\left(\left(\omega \cdot \rho_{c} / \eta_{c}\right)^{n} \exp \left(\left(-\omega-\operatorname{Int}_{g}\right) \cdot \rho_{c} / \eta_{c}\right) / n !\right) \cdot \exp \left(-\left(\operatorname{Int}_{E}+\operatorname{Int}_{L}\right) \cdot A-\operatorname{Int}_{E} \cdot \rho_{c} / \eta_{c}\right),
\end{aligned}
$$

其中

$$
A=|C| / \eta_{0}+\psi_{d c} \sum_{i=1}^{\max }\left(|C| \int_{\operatorname{dccmax}-i}^{\mathrm{dccmax}-i+1}\left(\exp \left(-\left(\ln (x)-\mu_{d c}\right)^{2} /\left(2 \sigma_{d c}^{2}\right)\right) /\left(x \sigma_{d c} \sqrt{2 \pi}\right)\right) \mathrm{d} x / \eta_{i}\right)
$$

\section{5 移动环境下不确定性组合服务的事务模型}

移动计算环境下, 受原子服务的可用性、可靠性和组合业务逻辑等影响, 组合服务成功率将产生 变化, 探讨不确定组合服务的事务处理理论与方法, 可以增强复杂服务组合的异常处理能力. 移动服 务组合可视为一个支持移动计算的业务服务流组合, 业务流中原子服务 (就组合事务而言, 即为任务 或子任务) 与移动计算环境中服务层的某类软件实体相关, 并通过后者的执行来实现. 基于移动组合 服务的事务类型, 在本文中简称移动组合事务. 下面给出移动组合服务具体描述:

定义 4 (移动组合服务) 移动组合服务定义为一个五元组 $\mathrm{TCS}=(I, \odot, \alpha, \beta, \gamma)$, 其中:

- $I=\left\{I_{1}, I_{2}, \ldots, I_{m}\right\}$, 其中 $I_{i} \in\left\{I^{p}, I^{c}, I^{r}, I^{v}\right\}(1 \leqslant i \leqslant m)$ 表示移动服务的任务.

- ○, 表示二元序列 $I_{i^{\circ}} I_{j}$, 其中 $o \in\{\prec, \varangle\} . I_{i} \prec I_{j}$ 表示强序, $I_{i}$ 先于 $I_{j}$ 执行; $I_{i} \varangle I_{j}$ 为弱序, 既不 满足 $I_{i} \prec I_{j}$, 也不满足 $I_{j} \prec I_{i}$.

- $\alpha: I \rightarrow$ Type 表示聚合映射函数, 其中 Type $\in$ \{null, sequence, And-join, Or-join, And-split, Orsplit, loop $\}$. 可用聚合 CS $:=\left(I_{i} \oplus I_{i+1}\right)\left|\left(I_{i}|| I_{i+1}\right)\right|\left(I_{i} \otimes I_{i+1}\right)\left|\left(I_{i} \Theta I_{i+1}\right)\right|\left\lfloor I_{i}\right.$ 形成更复杂的组合.

- $\beta: I \rightarrow$ State 表示状态映射函数, 其中 State $\in\{$ Ready, Activated, Running, Failed, Aborted, Cancelled, Committed, Compensated, Half_Compensated $\}$, 有 CS.state $=\cup_{i=1}^{n}\left(I_{i}\right.$. state $)$.

- $\gamma: I \rightarrow$ TransactionProperty 表示事务映射函数, 其中 TransactionProperty $\in\{$ Vital, Retriable, Compensable, Pivot $\}$, 有移动组合事务性质 CS.TransactionProperty $=\cup_{i=1}^{n}\left(I_{i}\right.$. TransactionProperty). 


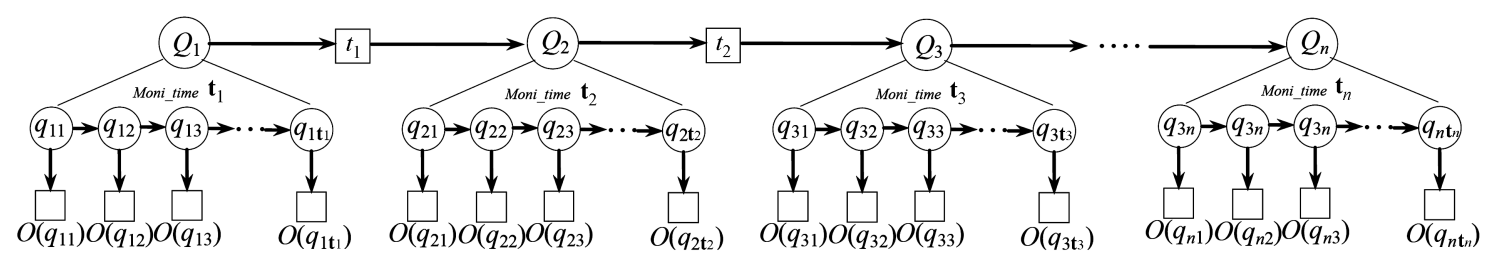

图 2 移动组合服务的 Markov 链模型

Figure 2 Markov chain model for mobile composition services

\section{1 支持移动组合服务的 Markov 模型}

在移动组合服务中, 除了原子服务的不确定性外, 组合服务任务在执行时, 表现出由任务的业务 逻辑变化而产生的不确定性, 即存在状态自发转移的不确定性, 用转移概率表征, 为了描述移动组合 服务的动态过程, 鉴于存在 9 种状态, 这里用 $\mathbf{P}_{i}^{(\mathbf{t})}=\left[\mathbf{P}_{1}^{(\mathbf{t})}, \mathbf{P}_{2}^{(\mathbf{t})}, \ldots, \mathbf{P}_{9}^{(\mathbf{t})}\right]$ 描述任务 $I$ (各逻辑单元) 在时刻 $\mathbf{t}$ 的状态转移概率分布, 用 $\mathbf{P}_{i}=\left[\mathbf{P}_{1}^{(0)}, \mathbf{P}_{2}^{(0)}, \ldots, \mathbf{P}_{9}^{(0)}\right]$ 表示任务 $I$ 的初始状态概率分布, 即 0 时刻的状态存在概率分布. 因此, 移动任务状态自发转移可用 Markov 链的转移过程来描述. 任务 $I$ 的执行受执行环境等不确定性因素影响, 可能产生状态转移, 此时的 $\mathbf{P}_{i}^{(\mathbf{t})}$ 将形成状态转移概率分 布, 用 $\pi=\left[\pi_{1}, \pi_{2}, \ldots, \pi_{9}\right]$ 表示, 则 $\pi_{i}=\mathbf{P}_{i}^{(\mathbf{t})}, i=1,2, \ldots, 9$. 基于隐 Markov 链构建状态转移模型 $\breve{S}=(\pi, \hat{A}, O)$, 初始概率分布矢量 $\pi=\left[\pi_{1}, \pi_{2}, \ldots, \pi_{9}\right]$, 其中 $\pi_{i}=\mathbf{P}_{i}^{(\mathbf{t})}, i=1,2, \ldots, 9 . \hat{A}$ 为状态转移矩 阵 $A_{N \times M}, O$ 为观测值矩阵 $O_{N \times M}$, 其中 $o_{i j}=O\left(q_{i j}\right)$, 它描述状态受外部影响情况. $N$ 为模型中状态 数数目, $M$ 为每个状态对应的可能观测值数.

Markov 链的基本方程 $\mathbf{P}_{i}^{(\mathbf{t}+1)}=\mathbf{P}_{i}^{(\mathbf{t})} \hat{A}$, 即 $\mathbf{P}_{i}^{(\mathbf{t}+1)}=\sum_{j=1}^{N} \mathbf{P}_{i}^{(\mathbf{t})} a_{i j}^{(\mathbf{t})}, i=1,2, \ldots, N, \mathbf{P}_{i}^{(\mathbf{t})}$ 分别为当 前状态概率分布 $\mathbf{P}_{i}^{(\mathbf{t})}=\mathbf{P}_{i}^{(0)} \hat{A}^{\mathbf{t}}$, 如 $\mathbf{P}_{i}^{(1)}=\sum_{j=1}^{N} \mathbf{P}_{i}^{(0)} a_{i j}^{(0)}, i=1,2, \ldots, N, \mathbf{P}_{i}^{(0)}$ 分别为初始状态概率分 布, 状态自发转移过程可通过状态转移矩阵 $\hat{A}$ 来控制.

移动组合服务的 Markov 链, 如图 2 所示 (其中 Moni_time 指观测时间), 可能经历了 $n$ 个状态 而具有 $Q_{1}, Q_{2}, \ldots, Q_{n}$ 共 $n$ 个状态观测值. 时刻 $q_{i}$ 记录其驻留时间内的细化 (如: $q_{i 1}, q_{i 2}, q_{i 3} \ldots$ ) 观 测值. 状态转移服从 Markov 性, 由转移概率 $\mathbf{P}\left(q_{i}\right)$ 决定, 其中 $\mathbf{P}\left(O\left(q_{n}\right) \mid\left(O\left(q_{1}\right)=o\left(q_{1}\right)\right) \wedge\left(O\left(q_{2}\right)=\right.\right.$ $\left.\left.o\left(q_{2}\right)\right) \wedge \cdots \wedge\left(O\left(q_{n-1}\right)=o\left(q_{n-1}\right)\right)\right)$ 定义了 $\mathbf{P}\left(q_{n} \mid q_{n-1}\right)$ 的一步转移概率, 描述从状态 $q_{n-1}$ 到状态 $q_{n}$ 的 概率. 若状态数为 $M$, 那么任务的一步转移概率矩阵 $\mathbf{P}$ 为 $\left[\begin{array}{ccc}\mathbf{P}_{11} & \cdots & \mathbf{P}_{1 M} \\ \vdots & & \vdots \\ \mathbf{P}_{N 1} & \cdots & \mathbf{P}_{N M}\end{array}\right]$, 其中 $\mathbf{P}_{i j} \geqslant 0$ 且每一行 $\sum \mathbf{P}_{i j}=1$. 用 $\mathbf{P}\left(q_{n+m} \mid q_{n}\right)$ 定义 $m$ 步转移概率, 记为 $\mathbf{P}^{(m)}$. 假定任务的初始状态概率矩阵为 $\mathbf{P}^{(0)}$, 则 $n$ 步后状态概率 $\mathbf{P}^{(n)}$, 其中 $\mathbf{P}^{(n)}=\mathbf{P}^{(0)} \mathbf{P}^{(n-1)}$, 这与常规 Markov 链状态和观测值一一对应不同. 由于 引入状态驻留时间, Markov 链中一个状态可能存在一段观测值, 因此 $Q_{i}$ 又可以划分为 $j$ 个子状态观 测值 $q_{i 1}, q_{i 2}, \ldots, q_{i j}$, 其中 $q_{i 1}$ 表示 $Q_{i}$ 的起始观测值, 驻留时间 $\mathbf{t}=q_{i j}-q_{i 1}$.

依据 Markov 链隐含状态和观测值之间的转移概率关系, 在状态转移模型 $\breve{S}$ 和观测状态序列 $Q_{i}$ 下, 计算任务状态观测序列 $O_{i}$ 的概率 $\mathbf{P}\left(O_{i} \mid Q_{i}, \breve{S}\right)=\mathbf{P}\left(o_{i 1} \mid q_{i 1}\right) \mathbf{P}\left(o_{i 2} \mid q_{i 2}\right) \ldots \mathbf{P}\left(o_{\text {in }} \mid q_{\text {in }}\right)=\prod_{j=1}^{n} \mathbf{P}\left(o_{i j} \mid q_{i j}\right)$.

若 Markov 链 $Q_{i}$ 及 $\breve{S}$ 确定时, 则可以计算出 $O$ 的概率分布. 先可计算动作状态序列 $Q_{i}$ 的概率分 布, $\mathbf{P}\left(Q_{i} \mid Q, \breve{S}\right)=\pi_{i} \prod_{j=1}^{i} \mathbf{P}\left(o_{j} \mid q_{j}, \breve{S}\right)$; 然后计算观测序列 $O$ 和状态序列 $Q_{i}$ 的联合概率 $\mathbf{P}\left(O, Q_{i} \mid \breve{S}\right)=$ $\mathbf{P}(O \mid Q, \breve{S}) \mathbf{P}\left(Q_{i} \mid \breve{S}\right)=\pi_{i} \prod_{j=1}^{i}\left(\mathbf{P}\left(o_{j} \mid q_{j}, \breve{S}\right) \mathbf{P}\left(q_{j}, \breve{S}\right)\right)$. 


\section{2 马氏模型中不确定性处理与特性分析}

\subsection{1 原子服务不确定性在模型中的传递}

在支持移动组合服务的 Markov 模型中, 各节点子任务对应的实施单元最终归结为原子服务, 各 移动原子服务藉助各自的接入方式影响组合服务, 其原子服务的可用与可靠属性也因此在该链式模型 中进行传递. 由于被组合原子服务的可用性和可靠性的不确定, 假设 WS 是组合服务中有限样本空间 中离散随机服务 $\mathrm{WS}_{i} \in\left\{\mathrm{WS}_{1}, \mathrm{WS}_{2}, \ldots, \mathrm{WS}_{n}\right\}$, 传递不确定性的数值表征即为可用性和可靠性概率的 组合运算. 基于本马氏模型的组合概率计算有: (1) 求和 $\sum_{i=1}^{n} \mathbf{P}\left(\mathrm{WS}_{i}\right) ;(2)$ 求积 $\Pi_{i=1}^{n} \mathbf{P}\left(\mathrm{WS}_{i}\right) ;(3)$ 条 件变量 $b_{i}$ 下的概率 $b_{i} \mathbf{P}\left(\mathrm{WS}_{i}\right) ;(4)$ 求最大值 $\max _{i \leqslant n} \mathbf{P}\left(\mathrm{WS}_{i}\right)$; (5) 求最小值 $\min _{i \leqslant n} \mathbf{P}\left(\mathrm{WS}_{i}\right)$. 典型情 况如下:

(1) 顺序聚合任务 $I_{\mathrm{seq}}=I_{1} \oplus I_{2} \oplus \cdots \oplus I_{n}$ (其中 $I_{i}$ 为 $\mathrm{WS}_{i}$ 的原子任务) 情况下, 聚合任务可用性为各 子任务可用性概率的乘积. 依据前述原子服务可用性公式 (4), $I_{i}$ 的可用性概率 $\mathbf{P}_{\mathrm{sa}}\left(\mathrm{WS}_{i}\right)=\mathbf{P}_{\mathrm{sa}}\left(P_{\mathrm{owi}}\right)$. $\mathbf{P}_{\mathrm{sa}}\left(C_{i}\right) . \mathbf{P}_{\mathrm{sa}}\left(E_{i}\right)$, 因此顺序聚合任务 $I_{\mathrm{seq}}$ 的可用性概率 $\mathbf{P}_{\mathrm{sa}}\left(I_{\mathrm{seq}}\right)=\Pi_{i=1}^{n}\left(\mathbf{P}_{\mathrm{sa}}\left(\mathrm{WS}_{i}\right)\right)=\Pi_{i=1}^{n}\left(\mathbf{P}_{\mathrm{sa}}\left(P_{\mathrm{owi}}\right)\right.$. $\left.\mathbf{P}_{\mathrm{sa}}\left(C_{i}\right) \cdot \mathbf{P}_{\mathrm{sa}}\left(E_{i}\right)\right)$. 同理, 依据前述原子服务可靠性公式 (9), 顺序聚合任务 $I_{\mathrm{seq}}$ 的可靠性概率

$$
\mathbf{P}_{\mathrm{sr}}\left(I_{\mathrm{seq}}\right)=\prod_{i=1}^{n}\left(\mathbf{P}_{\mathrm{sr}}\left(\mathrm{WS}_{i}\right)\right)=\prod_{i=1}^{n}\left(\mathbf{P}_{\mathrm{sr}}\left(\mathrm{cpu}_{i}\right) \cdot \mathbf{P}_{\mathrm{sr}}\left(\operatorname{mem}_{i}\right) \mathbf{P}_{\mathrm{sr}}\left(C_{i}\right) \cdot \mathbf{P}_{\mathrm{sr}}\left(E_{i}\right)\right) .
$$

(2) 并行聚合模式 $I_{\mathrm{par}}=I_{1}\left\|I_{2}\right\| \cdots \| I_{n}$ (其中 $I_{i}$ 为 $\mathrm{WS}_{i}$ 的原子任务) 情况下, 聚合任务不确定性 为各子任务相关概率的最大值. 基于前述原子服务不确定性公式 (4)(9), 并行聚合任务 $I_{\mathrm{par}}$ 的可用性 概率 $\mathbf{P}_{\mathrm{sa}}\left(I_{\mathrm{par}}\right)=\max _{i \leqslant n}\left(\mathbf{P}_{\mathrm{sa}}\left(\mathrm{WS}_{i}\right)\right)=\max _{i \leqslant n}\left(\mathbf{P}_{\mathrm{sa}}\left(P_{\mathrm{owi}}\right) \cdot \mathbf{P}_{\mathrm{sa}}\left(C_{i}\right) \cdot \mathbf{P}_{\mathrm{sa}}\left(E_{i}\right)\right)$. 同理, 并行聚合任务 $I_{\mathrm{par}}$ 的 可靠性概率 $\mathbf{P}_{\mathrm{sr}}\left(I_{\mathrm{par}}\right)=\max _{i \leqslant n}\left(\mathbf{P}_{\mathrm{sr}}\left(\mathrm{WS}_{i}\right)\right)=\max _{i \leqslant n}\left(\mathbf{P}_{\mathrm{sr}}\left(\mathrm{cpu}_{i}\right) \cdot \mathbf{P}_{\mathrm{sr}}\left(\operatorname{mem}_{i}\right) \cdot \mathbf{P}_{\mathrm{sr}}\left(C_{i}\right) \cdot \mathbf{P}_{\mathrm{sr}}\left(E_{i}\right)\right)$.

(3) 选择聚合模式 $I_{\mathrm{sel}}=I_{1} \otimes I_{2} \otimes \cdots \otimes I_{n}$ (其中 $I_{i}$ 为 $\mathrm{WS}_{i}$ 的原子任务, 条件变量 $b_{i} \in\{0,1\}$, $b_{i}=1$ 表示选择分支 $I_{i}$ 执行, 否则未被选中) 情况下, 聚合任务的不确定性为各子任务中被选中者的 相关概率. 基于前述原子服务不确定性公式 (4) 和 (9), 选择聚合任务 $I_{\mathrm{sel}}$ 的可用性概率 $\mathbf{P}_{\mathrm{sa}}\left(I_{\mathrm{sel}}\right)=$ $\Pi_{i=1}^{n}\left(b_{i} \cdot \mathbf{P}_{\mathrm{sa}}\left(\mathrm{WS}_{i}\right)\right)=\Pi_{i=1}^{n}\left(b_{i} \cdot \mathbf{P}_{\mathrm{sa}}\left(P_{\mathrm{owi}}\right) \cdot \mathbf{P}_{\mathrm{sa}}\left(C_{i}\right) \cdot \mathbf{P}_{\mathrm{sa}}\left(E_{i}\right)\right)$. 同理, 选择聚合任务 $I_{\mathrm{sel}}$ 的可靠性概率 $\mathbf{P}_{\mathrm{sr}}\left(I_{\mathrm{sel}}\right)$ $=\Pi_{i=1}^{n}\left(b_{i} \cdot \mathbf{P}_{\mathrm{sr}}\left(\mathrm{WS}_{i}\right)\right)=\prod_{i=1}^{n}\left(b_{i} \cdot \mathbf{P}_{\mathrm{sr}}\left(\mathrm{cpu}_{i}\right) \cdot \mathbf{P}_{\mathrm{sr}}\left(\mathrm{mem}_{i}\right) \cdot \mathbf{P}_{\mathrm{sr}}\left(C_{i}\right) \cdot \mathbf{P}_{\mathrm{sr}}\left(E_{i}\right)\right)$.

(4) 迭代聚合模式 $I_{\mathrm{itr}}=I_{i}$ (其中 $I_{i}$ 为 $\mathrm{WS}_{i}$ 的原子任务, $v$ 为迭代次数) 情况下, 聚合任务不确定 性为各子任务相关概率的迭代值. 基于前述原子服务不确定性公式 (4) 和 (9), 迭代聚合任务 $I_{\mathrm{itr}}$ 的可 用性概率 $\mathbf{P}_{\mathrm{sa}}\left(I_{\mathrm{itr}}\right)=\left(\mathbf{P}_{\mathrm{sa}}\left(\mathrm{WS}_{i}\right)\right)^{v}=\left(\mathbf{P}_{\mathrm{sa}}\left(P_{\mathrm{owi}}\right) \cdot \mathbf{P}_{\mathrm{sa}}\left(C_{i}\right) \cdot \mathbf{P}_{\mathrm{sa}}\left(E_{i}\right)\right)^{v}$. 同理, 迭代聚合任务 $I_{\mathrm{itr}}$ 的可靠性概 率 $\mathbf{P}_{\mathrm{sr}}\left(I_{\mathrm{itr}}\right)=\left(\mathbf{P}_{\mathrm{sr}}\left(\mathrm{WS}_{i}\right)\right)^{v}=\left(\mathbf{P}_{\mathrm{sr}}\left(\mathrm{cpu}_{i}\right) \cdot \mathbf{P}_{\mathrm{sr}}\left(\mathrm{mem}_{i}\right) \cdot \mathbf{P}_{\mathrm{sr}}\left(C_{i}\right) \cdot \mathbf{P}_{\mathrm{sr}}\left(E_{i}\right)\right)^{v}$.

\subsection{2 移动组合服务马氏模型中的转移聚合}

移动组合服务是由结构化的子任务服务组成, 任何组合服务都可以进行结构化解析. 显然移动组 合服务提供 CS : : =(I $\left.\oplus I_{i+1}\right)\left|\left(I_{i}|| I_{i+1}\right)\right|\left(I_{i} \otimes I_{i+1}\right)\left|\left(I_{i} \Theta I_{i+1}\right)\right|\left\lfloor I_{i}\right.$ 组合, 支持顺序、并行、选择和迭代 等聚合, 然而它们皆能以顺序与互斥等为基础完成聚合. 其中, 顺序与互斥结构可传递服务本身的不确 定性, 并可计算组合服务中业务逻辑的转移概率. 鉴于原子服务的不确定性传递已经讨论, 在此介绍基 于业务流解析的转移概率聚合情况.

(1) 顺序聚合 $(\odot)$ : 顺序聚合 $\mathrm{CS}=I_{1} \oplus I_{2} \oplus \ldots \oplus I_{n}$, 其中 $I_{i} \oplus I_{j}(1 \leqslant i, j \leqslant n)$ 满足 before $\left(t_{i}, t_{j}\right)$ 时 序, 且 $i<j, I_{i} \oplus I_{j}$ 成功执行时先激活 $I_{i}$ 再激活 $I_{j}, I_{j}$ 失败又影响 $I_{i}$. 假设 $I_{1}$ 和 $I_{2}$ 点火概率分别为 $\lambda_{1}$ 和 $\lambda_{2}$, 状态观测值分别为 $q_{1}$ 和 $q_{2}$, 则 $I_{1} \odot I_{2}$ 的观测值 $O\left(q_{1} \odot q_{2}\right)=O\left(q_{1}\right)+O\left(q_{2}\right)$, 设观测值 $O\left(q_{2}\right)$ 为 $\mathbf{t}$, 那么转移概率分布为 $\mathbf{P}\left(\left(O\left(q_{1}\right)+O\left(q_{2}\right)\right)<x\right)=\mathbf{P}\left(O\left(q_{1}\right)<x-O\left(q_{2}\right)\right)=1-\mathrm{e}^{-\lambda_{1}(x-\mathbf{t})}$. 
(2) 互斥聚合 $(\otimes)$ : 互斥聚合 $\mathrm{CS}=I_{1} \otimes I_{2}, I_{1}$ 与 $I_{2}$ 仅有分支之一成功执行. 假设 $I_{1}$ 和 $I_{2}$ 点火延迟 概率分别为 $\lambda_{1}$ 和 $\lambda_{2}$, 状态观测值分别为 $q_{1}$ 和 $q_{2}$, 转移权重分别为 $w_{1}$ 和 $w_{2}$, 且有 $w_{1}+w_{2}=1$, 权值比 $w=w_{2} / w_{1}$, 则 $I_{1} \otimes I_{2}$ 的观测值 $O\left(q_{1} \otimes q_{2}\right)=w_{1} . O\left(q_{1}\right)+w_{2} . O\left(q_{2}\right)$, 那么转移概率分布为 $\mathbf{P}\left(w_{1} \cdot O\left(q_{1}\right)+\right.$ $\left.w_{2} . O\left(q_{2}\right)<x\right)=\mathbf{P}\left(O\left(q_{1}\right)<x-w \mathrm{e}^{-\lambda_{i} x} O\left(q_{2}\right)\right)=1-\mathrm{e}^{-\lambda_{1} w(x-\mathbf{t})}$.

\subsection{3 移动组合服务马氏模型的特性分析}

基于 Markov 链实现移动组合服务的模型表达, 不仅可以便利地实现原子服务不确定性融合与传 递, 基于组合服务业务逻辑的转移概率表达, 同时具备无后效性、齐次性、稳定性特性.

(1) 无后效性. 移动组合服务在 $Q_{i}$ 时刻所处的状态条件, 与 $Q_{i}$ 时刻之前所处的状态条件具有无 关性, 即服务将来产生的状态仅与现在有关, 与过去的状态无关, 称为移动组合服务的无后效性. 若移 动组合服务执行的条件概率分布函数满足 $\mathbf{P}\left(O\left(q_{n}\right) \mid\left(O\left(q_{1}\right)=o\left(q_{1}\right)\right) \wedge\left(O\left(q_{2}\right)=o\left(q_{2}\right)\right) \wedge \cdots \wedge\left(O\left(q_{n-1}\right)=\right.\right.$ $\left.\left.o\left(q_{n-1}\right)\right)\right)=\mathbf{P}\left(O\left(q_{n}\right) \mid\left(O\left(q_{n-1}\right)=o\left(q_{n-1}\right)\right)\right)$, 称该过程为无后效的 Markov 过程. 也即在某时 $\mathbf{t}$ 从状态观 测值 $o\left(q_{i}\right)$ 转移到状态观测值 $o\left(q_{j}\right)$. 设转移步数为 $\ell$, 其无后效转移概率 $\mathbf{P}^{(j)}$ 满足 $\mathbf{P}^{(j)}=\mathbf{P}^{(i)} \mathbf{P}^{(j-i)}$. 由于组合服务执行状态是清晰的, $\ell$ 步转移概率是可确定值, 仍认为 $\mathbf{t}+m$ 时刻及以前所处的状态 与 $\mathbf{t}$ 时刻以后的状态 (包括失败恢复状态转移) 转移无关, 因此这里将带状态和时间的移动服务组合 Markov 过程视为无后效的 Markov 链.

(2) 齐次性. 若移动组合服务执行时状态间是无后效的, 且转移概率 $\mathbf{P}\left(O\left(q_{k+m}\right) \mid O\left(q_{k}\right)\right)$ 仅与 $q_{k}$ 和 $q_{k+m}$ 的时间间距相关, 那么 $\mathbf{P}\left(O\left(q_{k+m}\right) \mid O\left(q_{k}\right)\right)$ 转移概率具有平稳性, 称该链为齐次 Markov 链. 其中 $\mathbf{P}\left(O\left(q_{k+m}\right) \mid O\left(q_{k}\right)\right)$ 为齐次 Markov 链的 $m$ 步转移概率, 在此用 $\mathbf{P}_{i j}(m)$ 表示齐次 Markov 链的 $m$ 步 转移矩阵, $j$ 表示各观测阶段中的时刻标示数.

$$
\mathbf{P}_{i j}(m)=\left[\begin{array}{ccccc}
\mathbf{P}_{11}(1) & \mathbf{P}_{12}(1) & \cdots & \mathbf{P}_{1 j}(1) & \cdots \\
\mathbf{P}_{21}(1) & \mathbf{P}_{22}(1) & \cdots & \mathbf{P}_{2 j}(1) & \cdots \\
\vdots & \vdots & & \vdots & \vdots \\
\mathbf{P}_{i 1}(1) & \mathbf{P}_{i 2}(1) & \cdots & \mathbf{P}_{i j}(1) & \cdots \\
\vdots & \vdots & & \vdots & \vdots \\
\mathbf{P}_{n 1}(1) & \mathbf{P}_{n 2}(1) & \cdots & \mathbf{P}_{n j}(1) & \cdots
\end{array}\right], \ldots,\left[\begin{array}{ccccc}
\mathbf{P}_{11}(m) & \mathbf{P}_{12}(m) & \cdots & \mathbf{P}_{1 j}(m) & \cdots \\
\mathbf{P}_{21}(m) & \mathbf{P}_{22}(m) & \cdots & \mathbf{P}_{2 j}(m) & \cdots \\
\vdots & \vdots & & \vdots & \vdots \\
\mathbf{P}_{i 1}(m) & \mathbf{P}_{i 2}(m) & \cdots & \mathbf{P}_{i j}(m) & \cdots \\
\vdots & \vdots & & \vdots & \vdots \\
\mathbf{P}_{n 1}(m) & \mathbf{P}_{n 2}(m) & \cdots & \mathbf{P}_{n j}(m) & \cdots
\end{array}\right]
$$

(3) 稳定性. 若移动组合服务的齐次 Markov 链的状态空间为 $P_{I}^{s}$, 转移概率 $\mathbf{P}\left(O\left(q_{k+m}\right) \mid O_{n}\left(q_{k}\right)\right)$ 有极限 $\lim _{m \rightarrow \infty} \mathbf{P}\left(O\left(q_{k+m}\right) \mid O\left(q_{k}\right)\right)=\pi_{i}$ (对应 $\left.Q_{i}\right), k=1,2, \ldots, m$, 称该 Markov 链是稳定的. 一个 稳定的 Markov 链, 从时间 $\mathbf{t}_{i}$ 处状态观测值 $o\left(q_{i}\right)$ 到 $\mathbf{t}_{j}$ 处状态观测值 $o\left(q_{j}\right)$ 的概率 $\mathbf{P}^{(i \rightarrow j)}$, 也就存 在 $\ell$ 步无后效稳定转移. 对于组合服务, 服务提供者和用户都处于移动执行状态, 随时间变化用户与 服务间的距离也会动态变化, 若组合服务按照转移概率矩阵 $\mathbf{P}_{i j}(m)$ 成功转移并到达 Committed 状 态, 那么转移概率 $\mathbf{P}\left(O\left(q_{k+m}\right) \mid O\left(q_{k}\right)\right)$ 是平稳的; 若执行超时失败并转移到 Failed 状态, 执行替代服务, 最后转到 Committed 状态, 那么概率 $\mathbf{P}\left(O\left(q_{k+m}\right) \mid O\left(q_{k}\right)\right)$ 也是平稳转移的; 若转移失败并到达 Failed 状态, 执行恢复, 消除失败影响并转到 Committed 或 Compensated, 那么转移概率 $\mathbf{P}\left(O\left(q_{k+m}\right) \mid O\left(q_{k}\right)\right)$ 也是平稳的. 最终组合服务转移到一致状态下, 存在状态转移概率分布 $\pi_{i}=\left[\pi_{i 1}, \pi_{i 2}, \pi_{i 3}, \ldots\right]$ 且满足 
$\sum_{j=1}^{m} \pi_{i j}=1$, 其中 $\pi_{i j}>0$. 由此可见服从稳定 Markov 链分布, 其转移矩阵 $\mathbf{P}_{i j}(m)$ 极限分布是

$$
\lim _{m \rightarrow \infty} \mathbf{P}_{i j}(m)=\left[\begin{array}{ccccc}
\pi_{11} & \pi_{12} & \cdots & \pi_{1 j} & \cdots \\
\pi_{21} & \pi_{22} & \cdots & \pi_{2 j} & \cdots \\
\vdots & \vdots & & \vdots & \vdots \\
\pi_{i 1} & \pi_{i 2} & \cdots & \pi_{i j} & \cdots \\
\vdots & \vdots & & \vdots & \vdots \\
\pi_{n 1} & \pi_{n 2} & \cdots & \pi_{n j} & \cdots
\end{array}\right] .
$$

总之, 齐次 Markov 链不论从开始状态, 还是执行其间, 其所处状态的转移概率将稳定在 $\pi_{i}$.

\section{3 基于 Markov 链的移动组合事务模型}

基于事务特性需求, 不同的移动组合服务类型要求所有或几乎所有子事务都成功完成; 在移动组 合事务中存在众多的业务子任务活动, 后者可能存在潜在不一致状态, 需要自动消除或者人工干预, 这 些都是服务事务机制必须面对和解决的问题. 由于组合事务内部逻辑的复杂性和松弛需求动态性, 移 动组合事务架构形式多样. 针对马氏移动组合服务模型, 本文拟讨论 4 种典型事务架构 (如图 3 所示), 并采用下述事务实施方法：(1) 基于马氏模型, 将原子服务不确定性概率和组合服务中子任务活动转 移概率归并考虑, 归并时依据马氏各聚合模式进行 (如上节所述), 服务可用性概率, 可靠性概率在点 火转移处直接与逻辑转移概率聚合 (乘, 最大或最小等), 以形成新的聚合可用性概率, 聚合可靠性概 率并沿业务流 $F$ 方向传递; (2) 设定事务失效 (含过程及最终) 的判断依据为: 点火转移概率或组合事 务状态的可用性低于阈值 (如可用性概率 $\mathbf{P}_{I a}=0$ ), 或组合事务状态的可靠性低于阈值 (如可靠性概率 $\left.\mathbf{P}_{I r}=0.5\right)$. 典型事务架构有:

(1) 无失效恢复架构: 无需面向失败与不可靠的恢复.

(2) 两阶段提交事务架构: 第一阶段依据功能需求并考虑可用性和可靠性要求, 完成移动组合服 务预留, 第二阶段进行移动组合服务执行, 若第二阶段移动组合事务中任一子事务 (如原子服务) 执行 失败或不可靠, 对该移动组合子事务进行重试 (允许选新服务重试).

(3) 补偿恢复事务架构: 采用上述两阶段提交事务架构的类似机制, 若第二阶段移动组合事务在 某子事务处执行失败或不可靠, 补偿已经提交的子事务, 消除影响.

（4）保留协议架构: 采用上述两阶段提交事务架构的类似机制, 若第二阶段移动组合事务执行失 败或不可靠, 针对失效子事务进行重试, 在依然不成功时进行补偿.

为了计算移动组合事务成功执行的概率和子事务处于不一致状态的概率, 下面引入支持该架构的 离散时间马尔科夫模型. 其中, $n$ 为观察期间参与执行任务的子事务个数为, $N$ 为移动组合事务中子 事务的总个数, $\mathbf{P}_{f}$ 为任务执行失效 (含可用性可靠性低于阈值情况) 的聚合概率, $\mathbf{P}_{c}$ 为补偿失效的聚 合概率. 下面分别讨论 4 种架构下的执行情况.

(1) 支持 Markov 链的无失效恢复架构的概率 $\Pi_{i=1}^{n}\left(1-\mathbf{P}_{f i}\right)$.

(2) 支持 Markov 链的两阶段提交移动组合事务架构. 该架构无失效执行的概率是 $\Pi_{i=1}^{n}\left(1-\mathbf{P}_{f i}\right)$, 首次失效中止的概率是 $\mathbf{P}_{f n} \Pi_{i=1}^{n-1}\left(1-\mathbf{P}_{f i}\right)$, 重试成功的概率是 $\mathbf{P}_{f n}\left(1-\mathbf{P}_{f n}\right) \Pi_{i=1}^{n-1}\left(1-\mathbf{P}_{f i}\right)$. 移动组合 事务成功 (含一次重试) 的概率为 $\Pi_{i=1}^{n}\left(1-\mathbf{P}_{f i}\right)+\mathbf{P}_{f n}\left(1-\mathbf{P}_{f n}\right) \Pi_{i=1}^{n-1}\left(1-\mathbf{P}_{f i}\right)$. 


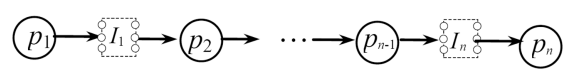

(a)

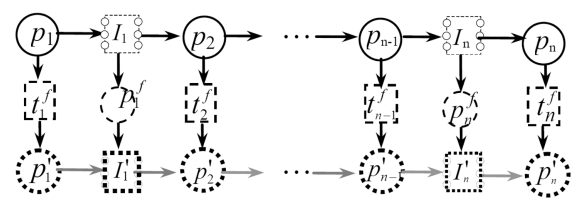

(b)

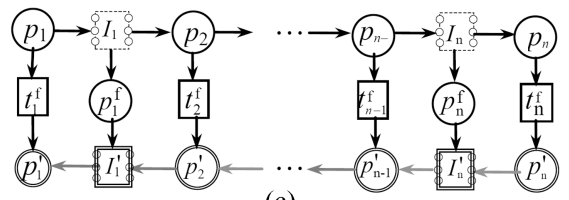

(c)

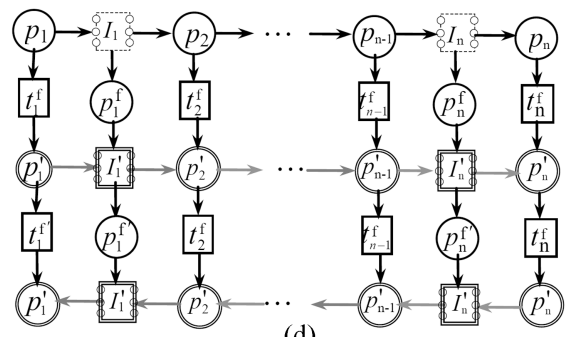

(d)

图 3 支持 Markov 模型的事务架构

Figure 3 Transaction patterns supported by Markov model. (a) Non failure recovery; (b) two-phase submission; (c) compensation recovery; (d) reserved protocol

(3) 支持 Markov 链的补偿恢复事务架构, 如果每个被调用的 WS 作为单个子事务处理, 当子事务 失效, 通过补偿事务来完成中止子事务. 移动组合事务无失效而成功执行的概率是 $\Pi_{i=1}^{n}\left(1-\mathbf{P}_{f i}\right)$, 移 动组合事务中第 1 个子事务首次失效的概率 $\mathbf{P}_{f 1}$, 第 1 个子事务首次补偿成功的概率 $\mathbf{P}_{f 1}\left(1-\mathbf{P}_{c 1}\right)$; 第 2 个子事务首次失效的概率 $\mathbf{P}_{f 2}$, 其首次补偿成功的概率 $\left(1-\mathbf{P}_{f 1}\right) \mathbf{P}_{f 2}\left(1-\mathbf{P}_{c 2}\right)\left(1-\mathbf{P}_{c 1}\right)$, 依次类 推, 第 $n$ 个子事务首次失效的概率 $\mathbf{P}_{f n}$, 前 $n$ 次补偿成功的概率 $\mathbf{P}_{f n} \Pi_{i=1}^{n-1}\left(1-\mathbf{P}_{f i}\right) \Pi_{j=1}^{n}\left(1-\mathbf{P}_{j}\right)$. 第 1 个子事务补偿后失效的概率 $\mathbf{P}_{f 1} \mathbf{P}_{c 1}$; 第 2 个子事务补偿后失效的概率 $\left(1-\mathbf{P}_{f 1}\right) \mathbf{P}_{f 2} \mathbf{P}_{c 2}$, 依此类推. 这里讨论的移动组合事务失效, 考虑补偿不成功则仅重试一次后的情况, 如果还存在不一致状态, 那 么需要人工干预. 若 $\mathbf{P}_{c}$ 值减少, 组合事务成功性增大.

(4) 支持 Markov 链的保留协议架构, 仅对移动组合事务中每个子事务单个重试, 但如果重试失 效, 则移动组合事务执行失效, 进行补偿且取消所有预留, 当然, 取消或预留都存在时限. 该架构模型 的移动组合事务无失效成功执行的概率是 $\Pi_{i=1}^{n}\left(1-\mathbf{P}_{f i}\right)$, 移动组合事务存在某个子事务之一执行失 效的概率 $\mathbf{P}_{f i}$, 重试后移动组合事务成功执行的概率是 $\mathbf{P}_{f n}\left(1-\mathbf{P}_{f n}\right) \Pi_{i=1}^{n-1}\left(1-\mathbf{P}_{f i}\right)$; 移动组合事务 允许 $m$ 个子事务执行失效 (后重试一次成功) 而其他 $n-m$ 个子事务一定执行成功的成功概率是 $\Pi_{i=1}^{n}\left(1-\mathbf{P}_{f i}\right)+\Pi_{j=1}^{m}\left(\left(1-\mathbf{P}_{f j}\right) P_{f j}\right) * \Pi_{k=m+1}^{n}\left(1-\mathbf{P}_{f k}\right)$.

\section{6 基于马氏模型的移动组合事务执行}

\section{1 移动组合事务的执行语义分析}

为了讨论移动组合服务的事务执行语义, 首先讨论原子任务的执行行为. 
(1) $\varsigma_{t_{i}} \mapsto\left(t_{i}, \square, u c_{i}, \widetilde{c}^{e s, c t_{i}}\right) \rightarrow\left(t_{i}, \diamond, u c_{i+1}, \widetilde{c}^{c m d, c t_{i+1}}\right), t_{i}$ 不确定执行并转入 Committed.

(2) $\varsigma_{t_{i}} \mapsto\left(t_{i}, \square, u c_{i}, \widetilde{c}^{e s, c t_{i}}\right) \rightarrow\left(t_{i}, \nabla, u c_{i+1}, \widetilde{c}^{f a l, c t_{i+1}}\right), t_{i}$ 不确定执行并转入 Failed.

(3) $\varsigma_{t_{i}} \mapsto\left(t_{i}, \square, u c_{i}, \widetilde{c}^{e s, c t_{i}}\right) \rightarrow\left(t_{i}, \perp, u c_{i+1}, \widetilde{c}^{a b t, c t_{i+1}}\right)$, 中止不确定 $t_{i}$ 执行, 转入 Aborted.

(4) $\varsigma_{t_{i}} \mapsto\left(t_{i}, \diamond, u c_{i}, \widetilde{c}^{e s, c t_{i}}\right) \rightarrow\left(t_{i}, \varangle, u c_{i+1}, \widetilde{c}^{c m p, c t_{i+1}}\right)$, 补偿不确定 $t_{i}$ 执行, 转入 Compensated.

(5) $\varsigma_{t_{i}} \mapsto\left(t_{i}, \nabla, u c_{i}, \widetilde{c}^{f a l, c t_{i}}\right) \rightarrow\left(t_{i}, \nabla_{\leftarrow}, u c_{i+1}, \widetilde{c}^{b r, c t_{i+1}}\right)$, 点火向后恢复.

(6) $\varsigma_{t_{i}} \mapsto\left(t_{i}, \nabla, u c_{i}, \widetilde{c}^{f a l, c t_{i}}\right) \rightarrow\left(t_{i}, \nabla_{\rightleftarrows}, u c_{i+1}, \widetilde{c}^{b f r, c t_{i+1}}\right)$, 点火向前恢复.

(7) $\varsigma_{t_{i}} \mapsto\left(t_{i}, \nabla, u c_{i}, \widetilde{c}^{f a l, c t_{i}}\right) \rightarrow\left(t_{i}, \nabla_{\uparrow}, u c_{i+1}, \widetilde{c}^{s r, c t_{i+1}}\right)$, 点火替代恢复.

令不确定状态 $\square \in\{\diamond, \nabla, \perp, \varangle\}$, 该集合元素分别对应状态: 成功、失效、终止与补偿. 依据任务 失效类型 $\left\{\nabla_{\leftarrow}, \nabla_{\rightleftarrows}, \nabla_{\uparrow}\right\}$ 点火失效恢复, $u c_{i}$ 描述不确定情境 (硬件、软件体或链路), $\widetilde{c}^{e, c t}$ 表示执行 的上下文; $\left(t_{i}, \square, u c_{i}, \widetilde{c}^{e s, c t t_{i}}\right)$ 描述 $t$ 执行语义转移, 可用 $\xi \mapsto c f g_{i} \rightarrow c f g_{i+1}$ 表达, 其中 $c f g_{i}$ 为初始格 局, $c f g_{i+1}$ 为转移格局. 对于 $I_{1} \odot I_{2} \odot \cdots \odot I_{n}, \odot \in\{\oplus, \|, \otimes, \Theta\}$, 从初始格局可能发生如下情况之一的 转移:

$$
\begin{aligned}
& \left\{\begin{array} { l } 
{ \varsigma _ { t _ { 1 } } \mapsto ( t _ { 1 } , \square , u c _ { 1 } , \widetilde { c } ^ { e s , c t _ { 1 } } ) } \\
{ \cdots } \\
{ \varsigma _ { t _ { i - 1 } } \mapsto ( t _ { i - 1 } , \square , u c _ { i - 1 } , \widetilde { c } ^ { e s , c t _ { i - 1 } } ) } \\
{ \varsigma _ { t _ { i } } \mapsto ( t _ { i } , \square , u c _ { i } , \widetilde { c } ^ { e s , c t _ { i } } ) } \\
{ \cdots } \\
{ \varsigma _ { t _ { n } } \mapsto ( t _ { n } , \square , u c _ { n } , \widetilde { c } ^ { e s , c t _ { n } } ) }
\end{array} \Rightarrow \left\{\begin{array} { l } 
{ ( t _ { 1 } , \diamond , u c _ { 2 } , \widetilde { c } ^ { c m d , c t _ { 2 } } ) } \\
{ \ldots } \\
{ ( t _ { i - 1 } , \diamond , u c _ { i } , \widetilde { c } ^ { c m d , c t _ { i } } ) } \\
{ ( t _ { i } , \diamond , u c _ { i + 1 } , \widetilde { c } ^ { c m d , c t _ { i + 1 } } ) } \\
{ \ldots } \\
{ ( t _ { n } , \diamond , u c _ { n + 1 } , \widetilde { c } ^ { c m d , c t _ { n + 1 } } ) }
\end{array} \text { or } \left\{\begin{array}{l}
\left(t_{2}, \nabla, u c_{2}, \widetilde{c}^{f a l, c t_{2}}\right) \\
\ldots \\
\left(t_{i}, \diamond, u c_{i}, \widetilde{c}^{c m d, c t_{i}}\right) \\
\left(t_{i+1}, \diamond, u c_{i+1}, \widetilde{c}^{c m d, c t_{i+1}}\right) \\
\ldots \\
\left(t_{n+1}, \square, u c_{n+1}, \widetilde{c}^{c m d, c t_{n+1}}\right)
\end{array}\right.\right.\right. \\
& \operatorname{or}\left\{\begin{array} { l } 
{ ( t _ { 2 } , \diamond , u c _ { 2 } , \widetilde { c } ^ { c m d , c t _ { 2 } } ) } \\
{ \ldots } \\
{ ( t _ { i } , \diamond , u c _ { i } , \widetilde { c } ^ { c m d , c t _ { i } } ) } \\
{ ( t _ { i + 1 } , \nabla , u c _ { i + 1 } , \widetilde { c } ^ { f a l , c t _ { i + 1 } } ) } \\
{ \ldots } \\
{ ( t _ { n + 1 } , \square , u c _ { n + 1 } , \widetilde { c } ^ { e , c t _ { n + 1 } } ) }
\end{array} \text { or } \left\{\begin{array} { l } 
{ ( t _ { 2 } , \diamond , u c _ { 2 } , \widetilde { c } ^ { c m d , c t _ { 2 } } ) } \\
{ \ldots } \\
{ ( t _ { i } , \diamond , u c _ { i } , \widetilde { c } ^ { c m d , c t _ { i } } ) } \\
{ ( t _ { i + 1 } , \perp , u c _ { i + 1 } , \widetilde { c } ^ { a b t , c t _ { i + 1 } } ) } \\
{ \ldots } \\
{ ( t _ { n + 1 } , \square , u c _ { n + 1 } , \widetilde { c } ^ { e , c o n t _ { n + 1 } } ) }
\end{array} , \text { 其中 } \left\{\begin{array}{l}
\left(t_{2}, \diamond, u c_{2}, \widetilde{c}^{c m d, c t_{2}}\right) \\
\ldots \\
\left(t_{i}, \diamond, u c_{i}, \widetilde{c}^{c m d, c t_{i}}\right) \\
\left(t_{i+1}, \nabla, u c_{i+1}, \widetilde{c}^{f a l, c t_{i+1}}\right) \\
\ldots \\
\left(t_{n+1}, \square, u c_{n+1}, \widetilde{c}^{e, c t_{n+1}}\right)
\end{array}\right.\right.\right.
\end{aligned}
$$

转移失败, 则点火恢复, 可能存在如下 3 种点火转移:

$$
\left\{\begin{array} { l } 
{ ( t _ { 2 } , \diamond , u c _ { 2 } , \widetilde { c } ^ { c m d , c t _ { 2 } } ) } \\
{ \ldots } \\
{ ( t _ { i } , \diamond , u c _ { i } , \widetilde { c } ^ { c m d , c t _ { i } } ) } \\
{ ( t _ { i + 1 } , \nabla _ { \leftarrow } , u c _ { i + 1 } , \widetilde { c } ^ { f a l , c t _ { i + 1 } } ) } \\
{ \ldots } \\
{ ( t _ { n } , \square , u c _ { n } , \widetilde { c } ^ { e , c t _ { n + 1 } } ) }
\end{array} \text { or } \left\{\begin{array} { l } 
{ ( t _ { 2 } , \diamond , u c _ { 2 } , \widetilde { c } ^ { c m d , c t _ { 2 } } ) } \\
{ \cdots } \\
{ ( t _ { i } , \diamond , u c _ { i } , \widetilde { c } ^ { c m d , c t _ { i } } ) } \\
{ ( t _ { i + 1 } , \nabla _ { \rightleftarrows } , u c _ { i + 1 } , \widetilde { c } ^ { f a l , c t _ { i + 1 } } ) } \\
{ \ldots } \\
{ ( t _ { n + 1 } , \square , u c _ { n + 1 } , \widetilde { c } ^ { e , c t _ { n + 1 } } ) }
\end{array} \text { or } \left\{\begin{array}{l}
\left(t_{2}, \diamond, u c_{2}, \widetilde{c}^{c m d, c t_{2}}\right) \\
\ldots \\
\left(t_{i}, \diamond, u c_{i}, \widetilde{c}^{c m d, c t_{i}}\right) \\
\left(t_{i+1}, \nabla_{\uparrow}, u c_{i+1}, \widetilde{c}^{f a l, c t_{i+1}}\right) \\
\ldots \\
\left(t_{n+1}, \square, u c_{n+1}, \widetilde{c}^{e, \text { cont }_{n+1}}\right)
\end{array} .\right.\right.\right.
$$

下面就两类基本情况 (顺序和互斥) 的失效执行语义进行分析

顺序执行情形如下 $\left(\right.$ ) 中所示. 顺序聚合 $I_{1} \oplus \cdots \oplus I_{n}$ 执行语义分析 $\oplus^{e}$, 若 $\oplus^{e}$ 正向执行处于 initial 和 final 的执行描述分别为 $\left\{\left(\oplus_{b}^{e}, f_{I i, i y}\right) \mid I_{i} \in \oplus^{e} \wedge i y \in \operatorname{in}\left(I_{i}\right)\right\}$ 和 $\left\{\left(\oplus_{b}^{e}, f_{I i, o y}\right) \mid I_{i} \in \oplus^{e} \wedge\right.$ oy $\left.\in \operatorname{out}\left(I_{i}\right)\right\}$; 若 $\oplus^{e}$ 逆向聚合恢复且处于 final 或 initial 的描述为 $\oplus_{r}^{r}=\left\{\left(r_{I i, i y}, \oplus_{b}^{r}\right) \mid I_{i}^{\prime} \in \oplus^{r} \wedge i y \in \operatorname{out}\left(I_{i}^{\prime}\right)\right\}$ 和 $\oplus_{r}^{f}=\left\{\left(\oplus_{b}^{r}, f_{I i, o y}\right) \mid\right.$ $I_{i}^{\prime} \in \oplus^{r} \wedge$ oy $\left.\in \operatorname{in}\left(I_{i}^{\prime}\right)\right\}$, 启动恢复处理器 $\operatorname{trigger}\left(I_{i}\right.$, invoke $\left._{I_{i}}^{\mathrm{RH}}\right) \Rightarrow\left(I_{c i}, \oplus_{\mathrm{Seq}}\right)$, 触发顺序恢复条件 getOp 
$\left(I_{i}^{\prime}, \oplus_{\text {Sequence }}\right)$ ? $\mathrm{OP}_{I^{\prime} i}: \operatorname{putprt}_{\oplus S e q}$, 由失败条件? 启动恢复策略 $\operatorname{get} \operatorname{Cpst}\left(I_{i}, r_{I i}, \oplus_{I i}, \widetilde{c}^{e, \alpha}\right)$, 并通过 binding $\left(I_{i}, I_{i}^{\prime}, r_{I_{i}}, r_{l_{i}}, \widetilde{c}^{e, \alpha}\right)$ 建立 $I_{i}$ 和 $I_{i}^{\prime}$ 之间映射; $\operatorname{rests}\left(I_{i}^{\prime}, \mathrm{HB}_{S^{\prime} I i}\right)$ 返回恢复结果.

$$
\left(\begin{array}{c}
\left(\operatorname{trigger}\left(I_{1}, \operatorname{invoke}_{I_{1}}^{\mathrm{RH}}\right) \wedge \operatorname{getCpst}\left(I_{1}, r_{I_{1, \oplus}}, \oplus_{I_{1}}, \widetilde{c}^{e, \alpha}\right) \wedge \operatorname{CHbegin}\left(I_{1}^{\prime}, \operatorname{HB}_{\oplus_{I_{1}}}\right)\right) \wedge \\
\ldots \\
\left(\operatorname{trigger}\left(I_{i}, \operatorname{invoke}_{I_{i}}^{\mathrm{RH}}\right) \wedge \operatorname{getCpst}\left(I_{i}, r_{I_{i, \oplus}}, \oplus_{I_{i}}, \widetilde{c}^{e, \alpha}\right) \wedge \operatorname{CHbegin}\left(I_{i}^{\prime}, \operatorname{HB}_{\oplus_{I_{i}}}\right)\right) \wedge \\
\ldots \\
\left(\operatorname{trigger}\left(I_{n}, \operatorname{invoke}_{I_{n}}^{\mathrm{RH}}\right) \wedge \operatorname{getCpst}\left(I_{n}, r_{I_{n, \oplus}}, \oplus_{I_{n}}, \widetilde{c}^{e, \alpha}\right) \wedge \operatorname{CHbegin}\left(I_{n}^{\prime}, \operatorname{HB}_{\oplus_{I_{n}}}\right)\right)
\end{array}\right)
$$

互斥情形如下 $\left(\right.$ ) 中所示互斥聚合 $I_{1} \otimes \cdots \otimes I_{n}$ 执行语义分析 $\otimes^{e}$, OR-split 执行映射 $O s \otimes_{I_{0}}^{f}$ $\times I_{i} \times O j \otimes_{I_{n+1}}^{s}$, 正向执行处于 final 和 initial 的 OR-join 分别描述为 $O j \otimes_{r}^{e}=\left\{\left(r_{I i, i y}, O j \otimes_{o z}^{e}\right) \mid I_{i} \in O j \otimes^{e}\right.$ $\left.\wedge i y \in \operatorname{in}\left(I_{i}\right) \wedge o z \in \operatorname{out}\left(I_{i}\right)\right\}$ 和 $O j \otimes_{f}^{e}=\left\{\left(O j \otimes_{I_{i}}^{e}, f_{I i, o y}\right) \mid I_{i} \in O j \otimes^{e} \wedge o y \in \operatorname{out}\left(I_{i}\right)\right\}$; 逆向聚合恢复处于 final 和 initial 的 OR-join 分别描述为 $O j \otimes_{r}^{r}=\left\{\left(r_{I i, i y} O j \otimes_{o z}^{r}\right) \mid I_{i} \in O j \otimes^{r} \wedge i y \in \operatorname{out}\left(I_{i}\right) \wedge o z \in \operatorname{out}\left(I_{i}\right)\right\}$ 和 $O j \otimes_{f}^{r}=\left\{\left(O j \otimes_{I_{i}}^{r}, f_{I i, o y}\right) \mid a x \in O j \otimes^{r} \wedge o y \in \operatorname{in}\left(I_{i}\right)\right\}$. 其中 $\operatorname{getOjh}\left(r_{I_{0}, i y}, O s \otimes_{I_{i}}^{s}\right)$ 获取互斥执行条件, skip (tokenbl(in $\left(I_{0}\right)$, sel $\left.)\right)$ 跳过互斥执行分支, evalbool(in $\left.\left(I_{0}\right)\right)$ 评价分支是否执行失败; 若执行失败启动类 似顺序执行恢复. 最后执行 getOjt $\left(O j \otimes_{I_{n+1}}^{s}, r_{I n+1, a w}\right)$ 完成 OR-join.

$$
\left(\begin{array}{c}
\operatorname{getOjh}\left(r_{I_{0}, a y}, O s \otimes_{I_{0}}^{s}\right) \wedge \operatorname{evalbool}\left(\operatorname{in}\left(I_{0}\right)\right) \wedge \operatorname{skip}\left(\operatorname{tokenbl}\left(\operatorname{in}\left(I_{0}\right), \operatorname{sel}\right)\right) \wedge \\
\left(\operatorname{trigger}\left(I_{1}, \operatorname{invoke}_{I_{1}}^{\mathrm{RH}}\right) \wedge \operatorname{getCpst}\left(I_{1}, r_{I_{1}, O S}, S_{I_{1}}, \widetilde{c}^{e, \alpha}\right) \wedge \operatorname{CHbegin}\left(I_{1}^{\prime}, \operatorname{HB}_{S_{I_{1}}}\right)\right) \vee \\
\ldots \\
\left(\operatorname{trigger}\left(I_{n}, \operatorname{invoke}_{A_{n}}^{\mathrm{RH}}\right) \wedge \operatorname{getCpst}\left(I_{n}, r_{I_{n}, O S}, S_{I_{n}}, \widetilde{c}^{e, \alpha}\right) \wedge \operatorname{CHbegin}\left(I_{n}^{\prime}, \operatorname{HB}_{S_{I_{n}}}\right)\right)
\end{array}\right)
$$

\section{2 移动组合事务的执行}

前一节主要讨论移动组合事务的执行语义分析, 然而在实际执行环境中, 移动组合事务还需考虑 上下文, 以此完成不确定性决策和事务一致性处理等具体动作. 下文将同样以顺序和互斥为例进行 说明.

若顺序聚合中任务是 compensable 或 nonvital, 某个任务执行失效, 中断顺序聚合执行, 其中对于 nonvital 情况, 由于其对成功不重要而不采取动作. 假定 $I_{i}$ 尝试用不同原子服务重试执行, 并点火前 向恢复, 令 $\boldsymbol{S}=I_{1} \oplus \cdots \oplus I_{n}$, 满足: (1) $I_{i} . \mathrm{TBP}=\mathrm{Vital} ;(2) I_{i}$.state=Executing. 下面讨论其不确定执行:

(1) $\zeta_{I 1 \oplus \cdots \oplus I n} \rightarrow\left(\boldsymbol{S}, \square, \tilde{c}^{{\text {cmd, } \text { cont }_{1}}_{1}}\right) \rightarrow\left(\boldsymbol{S}, \diamond, \tilde{c}^{\text {cmd, }^{2} \text { cont }} t_{n+1}\right)$, 任意 $I_{i}$. Committed 提交成功, 则 $\boldsymbol{S}$ 提交 成功, 执行继续.

(2) $\zeta_{I 1 \oplus \cdots \oplus I n} \rightarrow\left(\boldsymbol{S}, \nabla, \tilde{c}^{f a l, \text { cont }_{1}}\right) \rightarrow\left(\boldsymbol{S}, \nabla, I_{1}, \tilde{c}^{b r, \text { cont }_{2}}\right), I_{1}$ 执行失败并转状态 Failed 触发恢复: (1) 若 $\operatorname{TBP}\left(I_{1}\right)=$ Retriable 且 $\boldsymbol{S}=\emptyset$, 则启动重试恢复; (2) 若 $I_{1}$ 存在同类任务 $I_{1}^{\prime}$, 启动替代恢复.

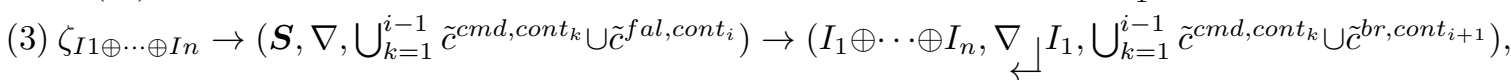
$I_{i}$ 执行失败, 如果 $\sigma_{1}$ 表示 $I_{1}, \ldots, I_{i-1}$, 且满足 $\forall I_{k} \mid\left(I_{k}\right.$.committed $) \in \sigma_{1} \wedge(1 \leqslant k \leqslant i)$, 可按情形 $(2)$ 的 两种恢复方式分别处理, 差别是 $\left(\boldsymbol{S}, \nabla, \tilde{c}^{f a l, c o n t_{i}}\right) \rightarrow\left(\boldsymbol{S}, \nabla_{t_{1} \cdots t_{i-1}} \mid t i, \tilde{c}^{b r, c^{\prime} o n t_{i+1}}\right)$, 若 $\boldsymbol{S} \neq \emptyset$ 且存在执行 迹 $\sigma_{1}$, 满足 $I_{i} . \mathrm{TBP} \in\{$ Vital, Compensable $\} \wedge\left(I_{i}\right.$. Committed $) \in \sigma_{1}$, 则逆向补偿恢复. 
(4) $\zeta_{S} \rightarrow\left(\boldsymbol{S}, \perp_{I i}, \bigcup_{k=1}^{i-1} \tilde{c}^{\text {cmd, cont }}{ }_{k} \cup \tilde{c}^{\text {abt, cont }}{ }_{i}\right)$, 当 $I_{i}$ 为 $I_{i}$.Running, $I_{1}, \ldots, I_{i-1}$ 均为 Committed, 向 $I_{i}$ 中止 Abort() 点火、补偿或重试 $I_{l} \in\left\{I_{1}, \ldots, I_{i-1}\right\}$ (其中 $1 \leqslant l \leqslant i-1$ ) 且 $I_{l}$.TBP $\in\{$ Compensable, Vital\}, 并启动逆向补偿恢复.

对于互斥情况, 令 $\boldsymbol{S}=I_{1} \otimes I_{2} \otimes \cdots \otimes I_{n}$. 若 $(\boldsymbol{S})_{\text {pre }}$ 的直接前驱为 $I_{m}$, 执行 $I_{m}$ 则激活 $\boldsymbol{S}$. 若 选择分支 $I_{i} \in S, I_{i}$ 是 vital 或 nonvital 的, 则 vital 终止于 committed, 而 nonvital 则为其他状态. 若 $I_{1} \otimes I_{2} \otimes \cdots \otimes I_{n}$ 之一满足 $I_{i} . \mathrm{TBP}=\mathrm{Vital}$, 则 $I_{i}$ 有效激活执行继续, 否则启动向后恢复; 若满足 $I_{i} . \mathrm{TBP}=\mathrm{ital} \wedge I_{m} . \mathrm{State}=$ committed, $I_{i}$ 有效激活. 如果提交失效或不可靠, 而 $I_{i}$ 是 nonCompensated, 则可能导致不一致性. 下面讨论不同点火条件下的互斥执行:

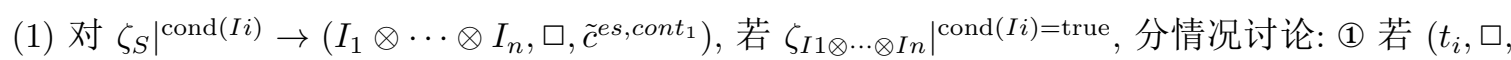
$\left.\tilde{c}^{e s, \text { cont }_{1}}\right)^{\text {true }} \rightarrow\left(t_{i}, \nabla, \tilde{c}^{f a l, \text { cont }_{1}}\right)^{\text {true }}$, 并 $I_{i}$ 处于 Failed, 启动恢复策略: (i) 若 $\boldsymbol{S}$ 仅涉及任务 $I_{i}$ 且 $I_{i}$. TBP

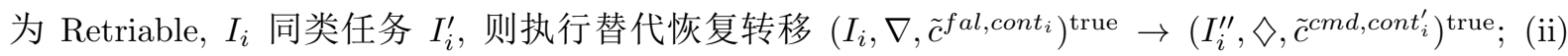
若 $\boldsymbol{S}$ 不为 $\square$, 且存在 $\sigma$, 满足 $\left(I_{i}\right.$. Committed $) \in \sigma \wedge I_{i}$.TBP $\in\{$ Compensable, Vital $\}$, 则启动逆向补偿 $\left(I_{i}, \nabla, \tilde{c}^{f a l, \text { cont }_{i}}\right)^{\text {true }} \rightarrow\left(I_{i}, \nabla_{\mid I_{i}}, \tilde{c}^{b r, \text { cont }_{i}}\right)^{\text {true }}$, 执行继续; (iii) 类似 (ii), 执行重试, 启动前向恢复; (2) 若 $\left(I_{1} \otimes \cdots \otimes I_{n}, \square, \tilde{c}^{e s, \text { cont }_{1}}\right) \rightarrow\left(I_{i}, \diamond, \tilde{c}^{c m d, \text { cont }_{i+1}}\right), I_{i}$ 执行成功, 执行继续.

(2) 对 $\left.\varsigma_{S}\right|^{\operatorname{cond}\left(I_{1}\right) \vee \cdots \vee \operatorname{cond}\left(I_{n}\right)=f a l s e}$, 若 case $\left(\operatorname{cond}_{1}\right) \vee \cdots \vee \operatorname{case}\left(\operatorname{cond}_{n}\right)$ 为 false, 则无互斥分支被激 活, 执行 $\operatorname{skip}\left(I_{1} \otimes I_{2} \otimes \cdots \otimes I_{n}\right)$ 绕过互斥分支.

\section{7 实验及其结果分析}

鉴于原子服务不确定性单独验证的不便, 本文拟将其融入组合服务的实验中, 从组合服务的执行 效果上来间接考查; 在组合服务中考虑服务可靠性和可用性进行事务处理, 以此分析和验证 4 种组合 事务恢复架构. 本文将以经典的旅行预订业务流程 (记为 TRP) 为例.

实验测试环境: 执行引擎为 ActiveBPEL, 选用有线与无线 (3G 和 Wifi 接入) 混合网络环境, 设 备有 2 台 $\mathrm{IBM} \times 3650$ 服务器 (4 核 Intel 2930, $1 \mathrm{G}$, SuSe Linux 10.2), 5 台有线网络终端 (Intel E5200, 2 G, Win XP SP2), 8 台无线接入笔记本电脑 (ThinkPad T440 i7-4510U, 4 G, Win7) 和 5 台移动智能 手机 (Sumsung N7100, 2 G, Android OS 4.1). 实验以 ActiveBPEL 为基础, 增加执行日志库和移动设 备环境感知信息数据库, 设计不确定性影响服务选择和事务失效判别机制, 扩展恢复处理器, 使其在 恢复中能重构恢复执行序列, 允许与外部事件进行交互, 允许子任务逻辑依赖.

实验中, 旅行者首先依据行程规划形成需求规格说明 (记为 CRS), 并以移动方式提交给旅行社; 依据 CRS, 随即旅行者和旅行社协商并确认旅行线路, 旅行社分别购买机票 (记为 FB) 或预订火车票 (记为 TR), 预订宾馆 (记为 $\mathrm{HB}$ ), 计算旅行目的地和酒店之间的距离, 向租赁小汽车 (记为 $\mathrm{CR}$ ) 或巴 士 (记为 $\mathrm{BR}$ ); 预订确认后, 旅行者执行移动在线支付 (记为 $\mathrm{OP}$ ); 选择快递公司 (记为 TDE、TDU) 投 递给旅行者, 将成功预订旅行发送至旅行者确认 (记为 TC). 上述任务流程由 10 多个子任务组成, 本 实验为一般事务规划 (含原子任务、替代任务和补偿任务). 设计实体任务个数 $n$ 达到 50 个, 而对于 保留协议架构实体任务数将是其他架构任务数的两倍, $n$ 将达到 100 个, 每个原子服务涉及两个保留 和确认/取消的子任务.

旅行预订流程 TRP 如图 4 所示. 本文将讨论聚合算子嵌套的旅行预订流程 $\mathrm{CRS} \oplus((\mathrm{FB} \otimes \mathrm{TR})\|\mathrm{HB}\|$ $(\mathrm{CR} \| \mathrm{BR})) \oplus \mathrm{OP} \oplus(\mathrm{TDE} \otimes \mathrm{TDU}) \oplus \mathrm{TC}$ 的可能事务行为. 对于 $\mathrm{TRP}$ 中任意 $I, I$ 的可能终止状态 $I . p^{s} \in$ \{aborted, canceled, failed, completed, compensated\}. 对于特定移动组合事务 TRP 的执行, 表 2 列出 


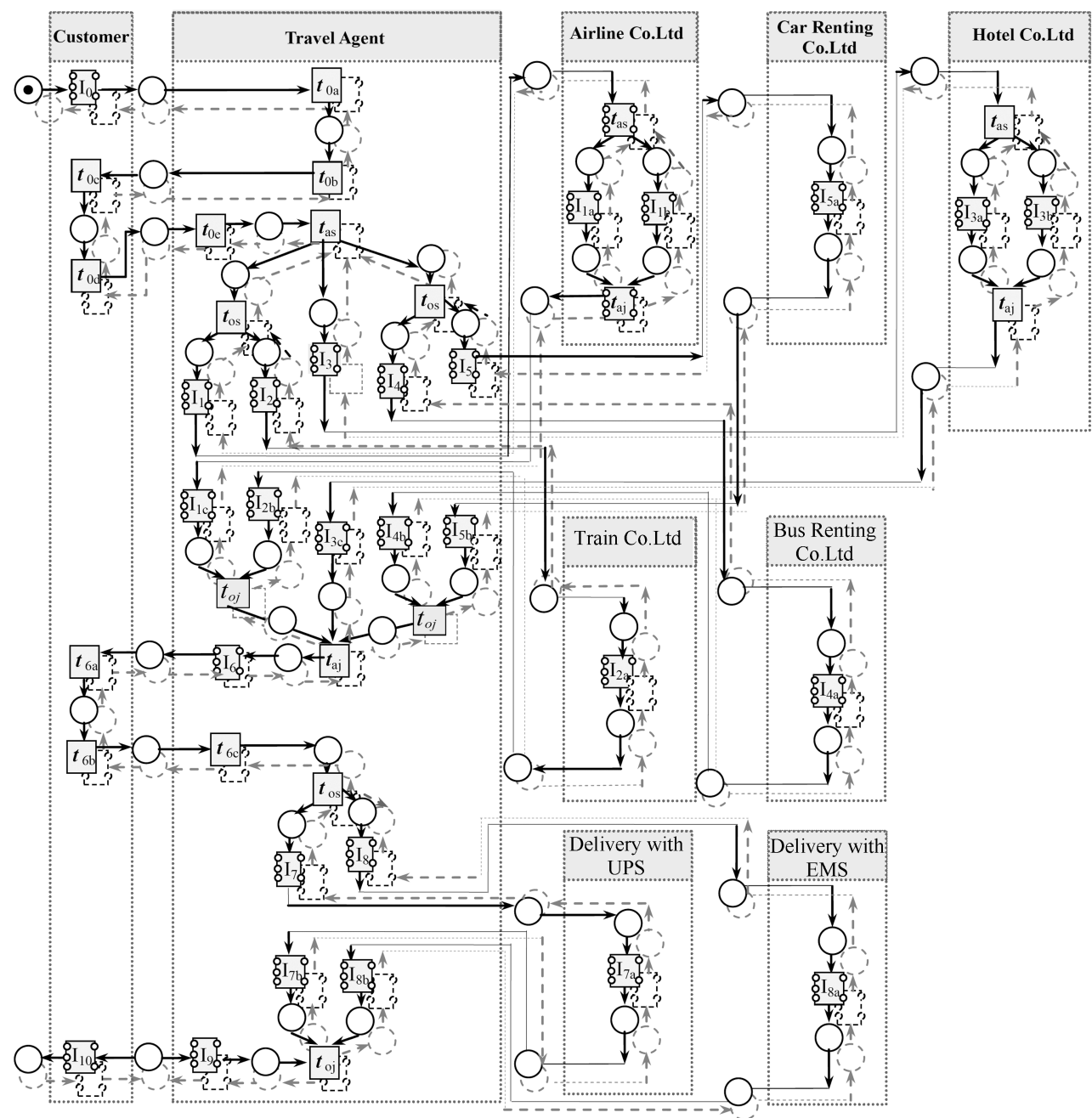

图 4 多伙伴协作旅行预订组合事务流程

Figure 4 A transactional workflow of TRP based on multi-partner cooperation

了 TRP 中可用服务 $\left\{w s_{0,1}, w s_{0,6}\right\},\left\{w s_{1,1}, w s_{1,4}\right\},\left\{w s_{2,2}, w s_{2,4}\right\},\left\{w s_{3,3}, w s_{3,4}, w s_{3,7}\right\},\left\{w s_{4,1}, w s_{4,3}\right\}$, $\left\{w s_{5,1}, w s_{5,4}, w s_{5,6}\right\},\left\{w s_{6,3}, w s_{6,5}\right\},\left\{w s_{7,1}, w s_{7,2}\right\},\left\{w s_{8,1}, w s_{8,3}\right\},\left\{w s_{9,1}, w s_{9,2}, w s_{9,3}\right\}$ 及其事务性质. 表 3 表示 TRP 的可接受状态集. 实验应用了补偿恢复和保留协议等架构, 以验证基于 Markov 链的 移动组合事务模型的合理性, 结果表明模型中应用的事务架构是可行的, 而且服务可靠性, 服务可用 性能为事务执行 (失效恢复和保留策略下的替换等) 提供了良好支持, 从而有效实现移动环境下事务 的一致性保持. 其中, 事务成功率作为关键指标, 它受到多种事务架构和服务不确定性因素影响, 相关 实验的详细数据及其分析如下.

为了考查高可用性可靠性情况下事务架构对事务成功率的影响, 实验首先构建一个稳定的良好服 务执行环境, 在广泛存在转移概率 (因业务逻辑) 的情况下, 选择具有同一事务目标的 4 类移动组合 服务流, 它们分别采用无失败恢复 (NFR)、两阶段提交架构 (TPS)、补偿恢复 (CR) 和保留协议 (RP) 等事务架构, 其参与总服务数限制在 200 内. 实验结果如图 5 所示. 在各子图中, $X$ 轴表示特定观察 时间段参与组合事务的服务个数 $m ; Y$ 轴表示自开始运行到某服务数时的积累事务成功概率. 图 5(a) 
表 2 可用服务及其事务性质

Table 2 Available services and their transaction properties

\begin{tabular}{|c|c|c|c|c|c|c|c|c|c|c|c|c|c|c|}
\hline Task & $w s$ & $\mathrm{TP}$ & Task & $w s$ & $\mathrm{TP}$ & Task & $w s$ & $\mathrm{TP}$ & Task & $w s$ & $\mathrm{TP}$ & Task & $w s$ & $\mathrm{TP}$ \\
\hline \multirow{8}{*}{ CRS } & $w s_{0,1}$ & $p$ & \multirow{7}{*}{ FB } & $w s_{1,1}$ & $c p$ & \multirow{7}{*}{$\mathrm{TR}$} & $w s_{2,1}$ & $c p$ & & $w s_{3,1}$ & $r$ & \multirow{7}{*}{$\mathrm{CR}$} & $w s_{4,1}$ & $r, c p$ \\
\hline & $w s_{0,2}$ & $r$ & & $w s_{1,2}$ & $r$ & & $w s_{2,2}$ & $p$ & & $w s_{3,2}$ & $r, c p$ & & $w s_{4,2}$ & $p$ \\
\hline & $w s_{0,3}$ & $r$ & & $w s_{1,3}$ & $r, c p$ & & $w s_{2,3}$ & $c p$ & & $w s_{3,3}$ & $p$ & & $w s_{4,3}$ & $r, c p$ \\
\hline & $w s_{0,4}$ & $p$ & & $w s_{1,4}$ & $p$ & & $w s_{2,4}$ & $r$ & HB & $w s_{3,4}$ & $c p$ & & $w s_{4,4}$ & $c p$ \\
\hline & $w s_{0,5}$ & $r$ & & $w s_{1,5}$ & $p$ & & $w s_{2,5}$ & $r, c p$ & & $w s_{3,5}$ & $p$ & & $w s_{4,5}$ & $p$ \\
\hline & $w s_{0,6}$ & $p$ & & $w s_{1,6}$ & $r, c p$ & & $w s_{2,6}$ & $p$ & & $w s_{3,6}$ & $c p$ & & $w s_{4,6}$ & $c p$ \\
\hline & $w s_{0,7}$ & $p$ & & $w s_{1,7}$ & $r$ & & $w s_{2,7}$ & $r, c p$ & & $w s_{3,7}$ & $c p$ & & & \\
\hline & $w s_{5,1}$ & $p$ & \multirow{6}{*}{$\mathrm{OP}$} & $w s_{6,1}$ & $p$ & \multirow{6}{*}{ TDE } & $w s_{7,1}$ & $r$ & \multirow{6}{*}{ TDU } & $w s_{8,1}$ & $p$ & \multirow{6}{*}{$\mathrm{TC}$} & $w s_{9,1}$ & $r$ \\
\hline \multirow{5}{*}{$\mathrm{BR}$} & $w s_{5,2}$ & $r, c p$ & & $w s_{6,2}$ & $r$ & & $w s_{7,2}$ & $p$ & & $w s_{8,2}$ & $c p$ & & $w s_{9,2}$ & $p$ \\
\hline & $w s_{5,3}$ & $c p$ & & $w s_{6,3}$ & $r$ & & $w s_{7,3}$ & $p$ & & $w s_{8,3}$ & $c p$ & & $w s_{9,3}$ & $p$ \\
\hline & $w s_{5,4}$ & $c p$ & & $w s_{6,4}$ & $p$ & & $w s_{7,4}$ & $r$ & & $w s_{8,4}$ & $c p$ & & $w s_{9,4}$ & $r$ \\
\hline & $w s_{5,5}$ & $r, c p$ & & & & & & & & $w s_{8,5}$ & $p$ & & & \\
\hline & $w s_{5,6}$ & $p$ & & & & & & & & $w s_{8,6}$ & $p$ & & & \\
\hline
\end{tabular}

表 3 可接受状态集

Table 3 Acceptable transation state sets

\begin{tabular}{|c|c|c|c|c|c|c|c|c|c|c|}
\hline ATS & CRS & FB & $\mathrm{TR}$ & НB & $\mathrm{CR}$ & $\mathrm{BR}$ & $\mathrm{OP}$ & TDE & TDU & $\mathrm{TC}$ \\
\hline$a t s_{1}$ & committed & committed & initial & committed & committed & initial & committed & initial & \multicolumn{2}{|c|}{ committed committed } \\
\hline$a t s_{2}$ & committed & committed & initial & committed & committed & initial & committed & initial & committed & failed \\
\hline$a_{t} s_{3}$ & committed & committed & initial & committed & committed & initial & committed & initial & failed & aborted \\
\hline ats $_{4}$ & committed & compensated & initial & compensated & compensated & initial & failed & aborted & aborted & aborted \\
\hline$a^{t} s_{5}$ & committed & compensated & initial & committed & cancelled & initial & failed & aborted & aborted & aborted \\
\hline$a^{t} s_{6}$ & committed & compensated & initial & committed & committed & initial & failed & aborted & aborted & aborted \\
\hline$a^{a t s_{7}}$ & committed & compensated & initial & cancelled & cancelled & initial & failed & aborted & aborted & aborted \\
\hline$a t s_{8}$ & committed & compensated & initial & committed & failed & aborted & aborted & aborted & aborted & aborted \\
\hline ats $s_{9}$ & committed & compensated & initial & cancelled & failed & aborted & aborted & aborted & aborted & aborted \\
\hline ats 10 & committed & compensated & initial & failed & aborted & aborted & aborted & aborted & aborted & aborted \\
\hline ats $_{11}$ & committed & failed & aborted & aborted & aborted & aborted & aborted & aborted & aborted & aborted \\
\hline$a^{a t s_{12}}$ & committed & initial & committed & committed & committed & initial & committed & initial & \multicolumn{2}{|c|}{ committed committed } \\
\hline$a^{a t s_{13}}$ & committed & initial & committed & committed & initial & \multicolumn{3}{|c|}{ committed committed committed } & initial & failed \\
\hline ats $_{14}$ & committed & initial & committed & committed & initial & \multicolumn{2}{|c|}{ committed committed } & failed & aborted & aborted \\
\hline ats $_{15}$ & committed & initial & compensated & cancelled & initial & committed & failed & aborted & aborted & aborted \\
\hline Ats $_{16}$ & committed & initial & compensated & committed & initial & cancelled & failed & aborted & aborted & aborted \\
\hline ats $_{17}$ & committed & initial & compensated & committed & initial & committed & failed & aborted & aborted & aborted \\
\hline$A t s_{18}$ & committed & initial & compensated & cancelled & initial & cancelled & failed & aborted & aborted & aborted \\
\hline Ats $_{19}$ & committed & initial & compensated & committed & aborted & failed & aborted & aborted & aborted & aborted \\
\hline ats $_{20}$ & committed & initial & compensated & cancelled & aborted & failed & aborted & aborted & aborted & aborted \\
\hline$A t s_{21}$ & committed & initial & compensated & failed & aborted & aborted & aborted & aborted & aborted & aborted \\
\hline$a^{a t s_{22}}$ & committed & initial & failed & aborted & aborted & aborted & aborted & aborted & aborted & aborted \\
\hline $\operatorname{ats}_{23}$ & committed & committed & failed & committed & committed & failed & committed & failed & committed & committed \\
\hline ats $_{24}$ & committed & failed & committed & committed & failed & committed & d committed c & committed & failed & committed \\
\hline
\end{tabular}



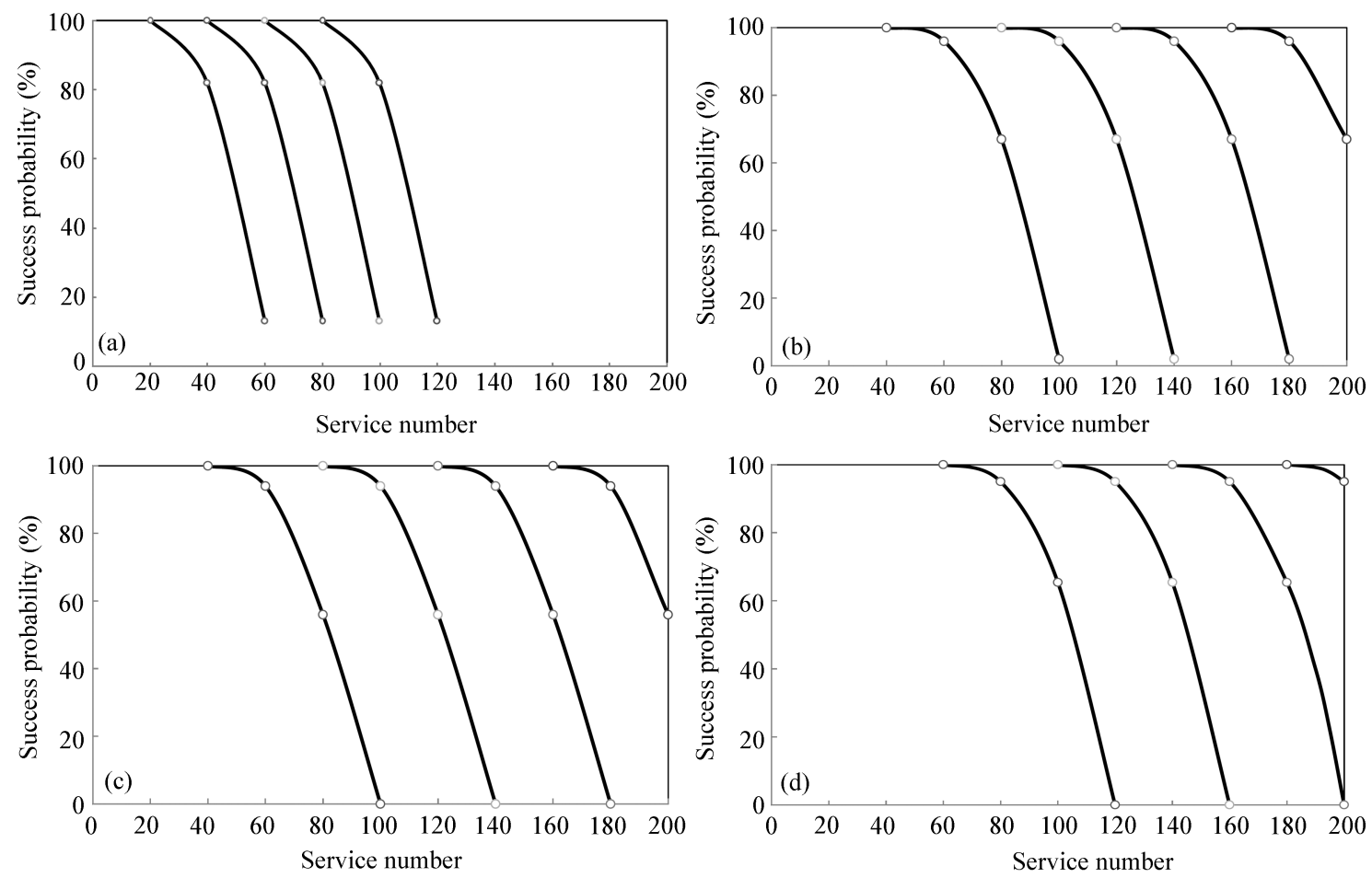

图 5 典型移动组合事务架构下事务执行成功概率

Figure 5 Transaction success probabilities under typical composition transaction patterns. (a) Non failure recovery; (b) two-phase submission; (c) compensation recovery; (d) reserved protocol

是 NFR 架构下的情况. 由于该架构无需尝试失效恢复, 故参与子服务数量少, 结果可见成功率下降很 快. 图 5(b) 显示了 TPS 架构下的情况. 由图知服务数越大, 单个任务重试成功的概率越高, 故总体成 功率则越大. 图 5(c) 是 CR 架构下的情况, 和 TPS 架构相比, 前者的执行成功率低于后者. 分析表明 补偿事务不同于正常执行程序, 难以规划和测试而出现性能下降, 它通常还涉及人工干预. 图 5(d) 是 RP 架构下的情况，曲线显示其具有较高可用性，且所涉服务数是前 3 种架构的两倍. 基于上述 4 个 情况来看预留协议架构虽对事务成功不具有完全保障性, 但相比其他架构具有更高可用性, 不过它将 会消耗更多预留资源.

为了探讨服务不确定性对组合事务成功的影响, 本文选择不同的不确定性指标阈值进行对比实验. 首先考查高可用性环境中不同可靠性阈值的服务选择参与的情况, 结果如图 6 中 (a) 和 (b) 所示; 对 高可靠性环境下的不同可用性阈值的情况则见图 6 中 (c) 和 (d). 两组实验皆选取具有同一事务目标 的两类移动组合服务流 (分别采用 RP 架构与 $\mathrm{CR}$ 架构, 参与服务数为 20), 其中组合事务中服务选择 的可靠性阈值与可用性阈值分别设定为 0.8 和 0.6. 由图 6 中执行曲线可发现: 服务选择时可靠性阈 值设定高的组合服务, 无论在哪种架构下其事务成功率都相对较高, 但在补偿事务架构下的可靠性阈 值越低成功率下降越快; 可用性阈值设定高的组合事务成功率高, 可用性阈值越低对成功率影响越大. 在保留协议架构下可靠性和可用性阈值变化对成功率影响相对平稳; 对照两种不确定性指标对成功率 的影响程度, 可用性比可靠性更大.

为了探讨服务流执行过程中, 各子任务阶段的服务聚合不确定性指标对当前组合事务的影响, 实 验选择不同架构下具有同一事务目标的同一组合服务流进行对比. 其中子任务由一组完成其子任务 

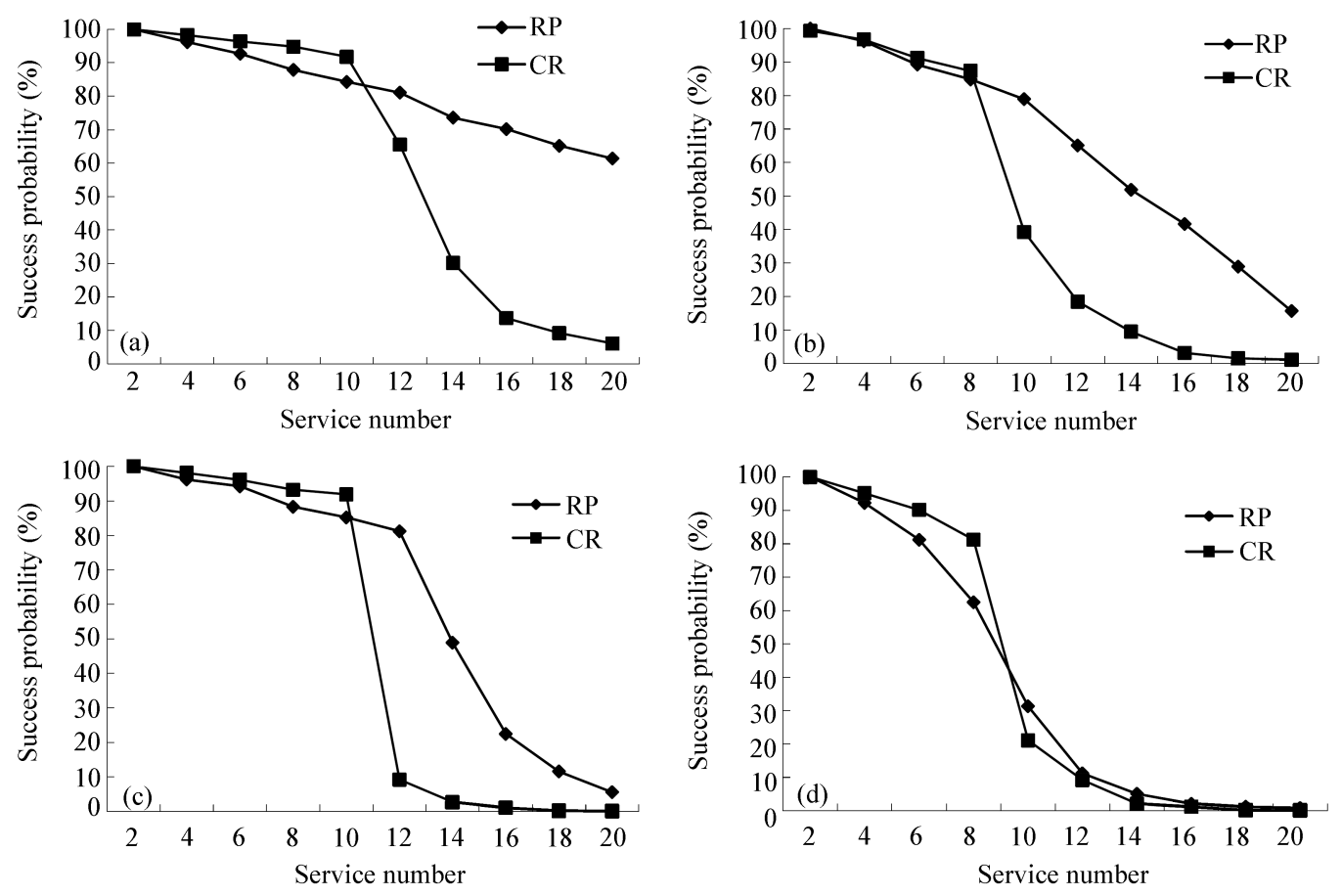

图 6 多不确定性阈值在移动组合事务架构下事务执行成功率比较

Figure 6 Transaction success probabilities with different thresholds of uncertainty. (a) Threshold of reliability=0.8; (b) threshold of reliability $=0.6$; (c) threshold of availability $=0.8$; (d) threshold of availability $=0.6$

目标的服务组执行完成; 如果子任务一次执行完成, 则该服务组仅需一个服务, 如果它由一个服务执 行但该服务发生了一次失败, 而后触发了一次补偿恢复, 并最后获得了执行成功, 则该组需要 2 个服 务 (即该服务及一个补偿恢复服务). 实验分别在 NFR 架构和 CR 架构下进行, 结果如图 7 所示. 其中 $X$ 轴表示运行到各子任务时, 子任务对应的服务组编号数; 图 7(a)(b)(c) 分别显示了一次实验的 3 个 考查指标情况, 其 $Y$ 轴分别指示自开始运行到该子任务时各指标的积累值, 即运行至对应服务组时的 事务聚合成功率, 服务聚合可靠性和服务聚合可用性. 对照 3 张图, 无论 NFR 还是 CR 中, 它们的事 务聚合成功率随聚合可靠性与聚合可用性同步降低, 且前者比后两者的值小; 前述结论中 CR 架构相 对 NFR 架构更明显; CR 架构下的 3 个指标值比 NFR 架构下的低, 并随着服务组数的增大, CR 架构 下 3 指标下降更快. 可见, 聚合成功率总是低于服务聚合不确定性指标, 且聚合不确定性指标对组合 事务架构敏感.

为了进一步明晰执行过程中服务聚合不确定性指标对当前组合事务的影响, 实验选择同一事务架 构 $(\mathrm{CR})$, 并具有同一事务目标的 5 组服务流. 结果如图 8 所示. 其中横坐标和纵坐标表示情况与图 7 相同, 图 $8(\mathrm{a}) \sim(\mathrm{c})$ 也是指示了同实验的 3 个考查指标情况. 由图 8 可知 5 组事务在执行中 3 个指标各 自有明显差异, 其共同特征是可靠性下降最快; 图 8(b) 和 (c) 中同一组服务流的聚合不确定性指标出 现拐点, 将促使图 8(a) 中同一组服务流的事务聚合成功率出现同类拐点, 如图 8(b) 中组 2 执行到第 6 和第 8 个服务时可靠性出现下行拐点, 则马上导致图 8(a) 中同组执行到对应服务处, 其事务聚合成 功率突然下降. 图 8(b) 和 (c) 中聚合性指标都高的组有组 2 和组 4, 但图 8(a) 中只有组 2 的成功率较 高. 分析表明: 同事务目标的同类组合服务流随参与执行服务的不同, 不确定性等可能出现较大差异, 且服务的可用性指标相对可靠性指标较为稳定; 服务不确定性指标出现拐点, 也将造成事务成功率在 

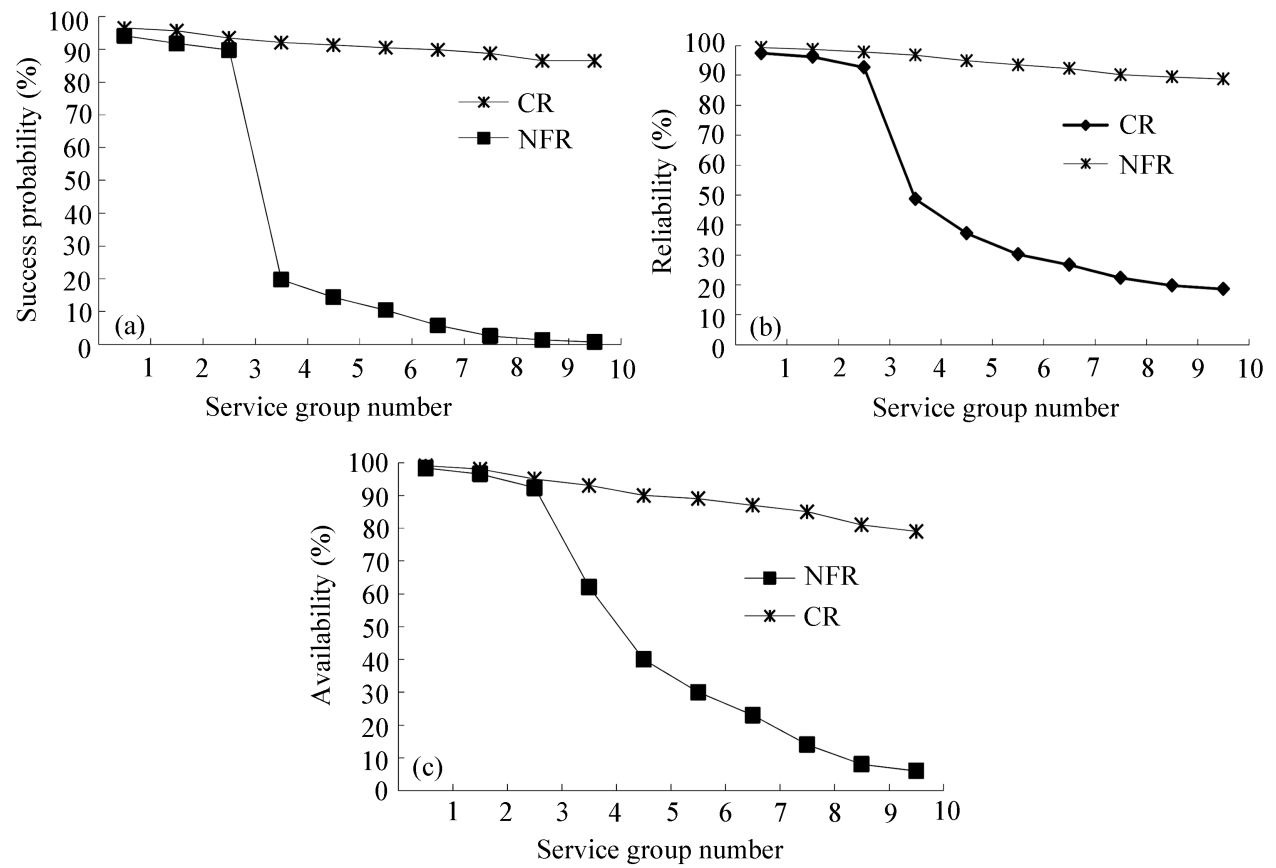

图 7 不同事务架构下聚合不确定性的变化及其对事务的影响

Figure 7 Aggregation uncertainty changes and their impact on transactions under different transaction patterns. (a) Aggregation success probabilities; (b) aggregation reliability probabilities; (c) aggregation availability probabilities

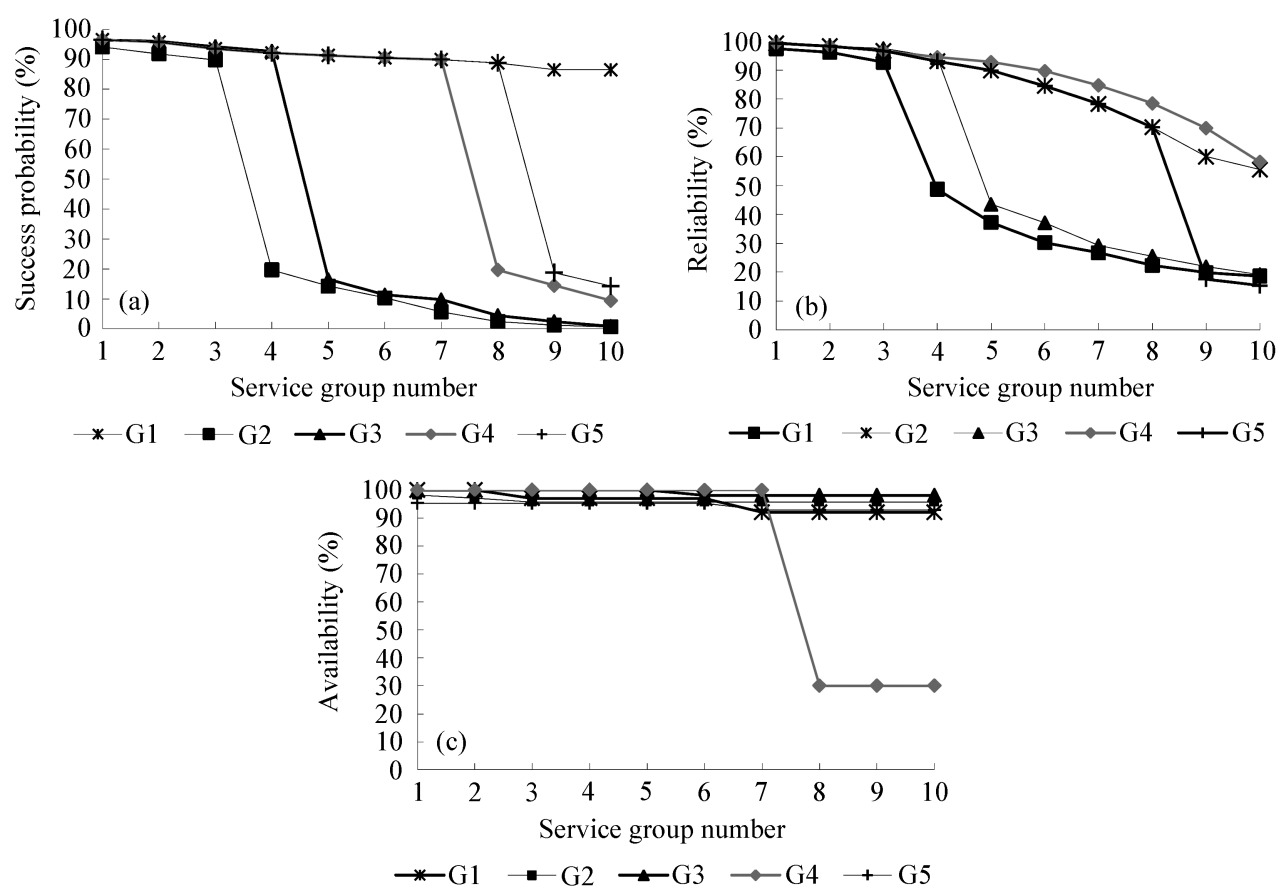

图 8 同事务架构下聚合不确定性的变化及其对事务的影响

Figure 8 Aggregation uncertainty changes and their impact on transactions under the same transaction pattern. (a) Aggregation success probabilities; (b) aggregation reliability probabilities; (c) aggregation availability probabilities 
对应处出现拐点; 服务不确定性指标都良好的情况下，也存在事务失败的可能性，这可能是基于业务 逻辑的转移概率等因素造成的.

\section{8 结论}

本文以移动环境下原子服务的不确定性表达和事务描述为基础, 提出了一种基于 Markov 链的移 动组合事务模型. 该模型能有效刻画事件点火转移和服务不确定性聚合, 同时能较好地实现典型事务 架构下的事务处理. 基于形式化方法的理论分析表明该模型下事务执行语义正确并可行. 以多伙伴协 作旅行预订服务为例, 实验验证移动组合事务模型在应用中具有有效性, 比较发现保留协议下的事务 成功率最高, 而且服务不确定性指标对事务执行影响显著.

下一步拟对现有移动组合事务模型中的多种关系进行多角度验证与分析, 对于部分情境因素, 采 用语义与概率混合描述方法, 增强组合事务模型的表达能力, 提高移动环境下不确定性组合服务的事 务成功率和实用性.

\section{参考文献}

1 Gorbenko A, Kharchenko V, Tarasyuk O, et al. The threat of uncertainty in service-oriented architecture. In: Proceedings of the 2008 RISE/EFTS Joint International Workshop on Software Engineering for Resilient Systems, New York, 2008. 49-54

2 Haddad J E, Manouvrier M, Rukoz M. TQoS: transactional and QoS-aware selection algorithm for automatic web service composition. IEEE Trans Serv Comput, 2010, 3: 73-85

3 Chu H H, You C W, Teng C M. Challenges: wireless web services. In: Proceedings of the 10th International Conference Parallel and Distributed Systems, Newport Beach, 2004. 657-664

4 Yang Z H, Liu C H. Implementing a flexible compensation mechanism for business processes in web service environment. In: Proceedings of International Conference on Web Services, Chicago, 2006. 753-760

5 Khachana R T, Jamesa A E, Iqbal R. Relaxation of ACID properties in AuTrA: the adaptive user-defined transaction relaxing approach. Fut Gener Comput Syst, 2011, 27: 58-66

6 Kokash N, Arbab F. Formal design and verification of long-running transactions with extensible coordination tools. IEEE Trans Serv Comput, 2013, 6: 186-200

7 Rajaram K, Babu C, Adiththan A. Specification of transactional requirements for web services using recoverability. Int J Inf Tech Web Eng, 2013, 8: 51-65

8 Yin Y Y, Li Y, Dend S G, et al. Determining on consistency and compatibility of web services behavior. Act Electron Sin, 2009, 37: 433-438 [殷昱显, 李莹, 邓水光, 等. Web 服务行为一致性与相容性判定. 电子学报, 2009, 37: 433-438]

9 Zhang X G, Liu H B, Abraham A. A novel process network model for interacting context-aware web services. IEEE Trans Serv Comput, 2013, 6: 344-357

10 Medjahed B, Malik Z. Bottom-up fault management in composite web services. Lect Notes Comput Sci, 2011, 6741: 597-611

11 Sun H Y, Yang J. Exploiting CoBTx-Net to verify the reliability of collaborative business transactions. In: Proceedings of the 2nd IEEE Asia-Pacific Service Computing Conference, Washington, 2007. 415-422

12 Sun H Y, Yang J. BTx-Net: a token based dynamic model for supporting consistent collaborative business transactions. Lect Notes Comput Sci, 2008, 4928: 220-231

13 Mei X Y, Huang C Q, Zheng X L, et al. Failure recovery algorithm for LRT and transaction property analysis. J Commun, 2012, 33: 31-41 [梅晓勇, 黄昌勤, 郑小林, 等. 支持 LRTs 的失败恢复算法及其事务性质分析. 通信学报, 2012, 33: 31-41]

14 Mei X Y, Li S X, Huang C Q, et al. An execution semantic analysis method for composition transaction. Act Electron Sin, 2012, 40: 1386-1396 [梅晓勇, 李师贤, 黄昌勤, 等. 一种支持组合事务的执行语义分析方法. 电子学报, 2012, 40: $1386-1396]$ 
15 Pezoa J E, Dhakal S, Hayat M M. Maximizing service reliability in distributed computing systems with random node failures: theory and implementation. IEEE Trans Parall Distr Syst, 2010, 21: 1531-1544

16 Akkarajitsakul K, Hossain E, Niyato D. Distributed resource allocation in wireless networks under uncertainty and application of bayesian game. IEEE Commun Mag, 2011, 49: 120-127

17 Zhang Y, Zhang W J, Lin Q L, et al. Effectively indexing the multidimensional uncertain objects. IEEE Trans Knowl Data Eng, 2014, 26: 608-622

18 Lee S K. Context modeling and inference system for heterogeneous context aware service. Lect Notes Comput Sci, 2007, 4558: 413-422

19 Christian K. Processing the uncertainty: quality-aware data stream processing for dynamic context models. In: Proceedings of 2012 IEEE International Conference on Pervasive Computing and Communications Workshops, Lugano, 2012. 560-561

20 Anagnostopoulos C B, Ntarladimas Y, Hadjiefthymiades S. Situational computing: an innovative architecture with imprecise reasoning. J Syst Softw, 2007, 80: 1993-2014

21 Mo T, Li W P, Wu Z H, et al. Framework of context-aware based service system. Chin J Comput, 2010, 33: 2084-2092 [莫同, 李伟平, 吴中海, 等. 一种情境感知服务系统框架. 计算机学报, 2010, 33: 2084-2092]

22 Wen S T, Tang C G, Li Q, et al. Probabilistic top-K dominating services composition with uncertain QoS. Serv Oriented Comput Appl, 2014, 8: 91-103

$23 \mathrm{Gu} \mathrm{J}$, Luo J Z, Cao J X, et al. Performance modeling and analysis for composite service considering failure recovery. J Softw, 2013, 24: 696-714 [顾军, 罗军舟, 曹㺵新, 等. 考虑失效恢复的组合服务性能建模与分析. 软件学报, 2013, 24: $696-714]$

\title{
Transaction modelling and execution analysis of uncertainty com- position service in mobility computing environments
}

\author{
HUANG ChangQin ${ }^{1,2 *}$, MEI XiaoYong ${ }^{1}$, ZHAO GanSen ${ }^{1}$, WU JiYi ${ }^{2,4}$, TANG Yong ${ }^{1}$, \\ WANG HuiJin ${ }^{3} \&$ ZHENG XiaoLin ${ }^{2}$
}

1 Research Center for Information Services and Software Technology, South China Normal University, Guangzhou 510631, China;

2 E-Service Research Center, Zhejiang University, Hangzhou 310027, China;

3 Department of Computer, Jinan University, Guangzhou 510632, China;

4 Key Lab of E-Business, Hangzhou Normal University, Hangzhou 310036, China

*E-mail: cqhuang@zju.edu.cn

\begin{abstract}
In mobile-enabled service-oriented computing, there exist resource restrictions and connection intermittences, and transactional services are often confronted with handovers and weak consistency support. In order to ensure the transaction validity of composition services in mobile environments, a mobile composition transaction model based on Markov Chain is proposed via analyzing service uncertainty and transaction properties. Firstly, formal methods are applied to construct a mobile atomic service model and a transaction mechanism of atomic services, and the service uncertainties are formulated with state probabilities. Secondly, the mobile composition service model is constructed using a Markov Chain, in which uncertainties are passed and a method of reliable composition transaction is presented. Then transaction execution of typical logic structure is introduced after execution semantic analysis. Finally, an application case shows that this model is effective to describe the mobile composition transaction, and the experimental results suggest that service availability, service reliability and transaction pattern have a considerable impact on the probability of success of composition transactions.
\end{abstract}

Keywords composition service, uncertainty dependency, Markov chain, transaction, execution analysis 


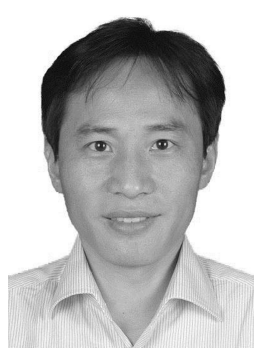

HUANG ChangQin was born in 1972. He received Ph.D. degree in computer science and technology from Zhejiang University, Hangzhou in 2005. Currently, he is a professor at South China Normal University (SCNU). His research interests include service computing, cloud computing, semantic web and education informationization. Prof. Huang is a member of CCF, ACM and IEEE.

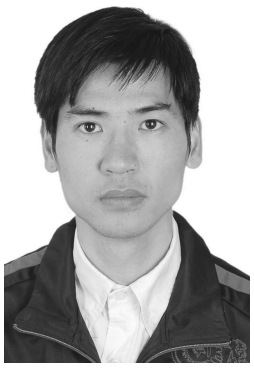

ZHAO GanSen was born in 1977. H received his Ph.D. degree in computer security from the University of Kent, UK in 2007. Currently, he is a professor at SCNU. His research interests include trust management, cloud computing, and massive data processing. $\mathrm{He}$ is also a member of China Big Data Expert committee.

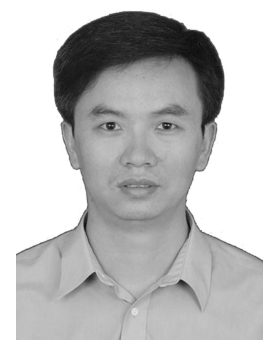

MEI XiaoYong was born in 1974. He received $\mathrm{Ph}$. D. degree in computer software and theory from Sun Yat-sen University, Guangzhou in 2011. Currently, he is a post-doctor at SCNU. His research interests include service computing, semantic web and Petri nets. Dr. Mei is a member of CCF.

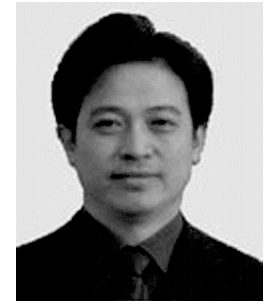

TANG Yong was born in 1964. $\mathrm{He}$ received $\mathrm{Ph} . \mathrm{D}$. degree in computer science and technology from University of Science and Technology of China, Hefei in 2001. Currently, he is a Professor at SCNU. His research interests include database, and collaboration computing. $\mathrm{He}$ is now a dean of School of Computer at SCNU and is a senior member of $\mathrm{CCF}$. 